\title{
Beam steering and coordinate system rotation improves accuracy of ultrasonic measurements of tissue displacement vector and lateral displacement
}

\author{
This article was published in the following Dove Press journal: \\ Reports in Medical Imaging \\ 14 November 2011 \\ Number of times this article has been viewed
}

\author{
Chikayoshi Sumi ${ }^{1,2}$ \\ Kento Ichimaru ${ }^{2}$ \\ Yusuke Shinozuka² \\ 'Department of Information and \\ Communication Science, ${ }^{2}$ Department \\ of Electrical and Electronics \\ Engineering, Faculty of Science and \\ Technology, Sophia University, \\ Kioi-cho, Chiyoda-ku, Tokyo, Japan
}

\begin{abstract}
With the proper use of beam steering and apodization, a higher resolution lateral echo image is obtained than with conventional imaging. This is achieved by superimposing crossed, steered beams, which is referred to as "lateral modulation" (LM). This type of beamforming achieves almost the same accuracy in lateral displacement measurements as in axial displacement measurements, ie, displacement vector measurements. The steering angle (ASTA) can also be used instead of LM, which uses only beams with a steering angle. In this report, the accuracy of the displacement vectors and lateral displacement measurements for LM and ASTA were evaluated using simulations and agar phantom experiments. The parameters used were direction of a displacement vector, steering angles, and rotation angle for the coordinate system. Changes in the steering angle and rotation angle of the coordinate system permit control of frequencies in the respective directions of the coordinate axes. As shown, when performing a simple ASTA for displacement vector measurement, a spectra frequency division should be performed using a previously developed multidimensional autocorrelation or Doppler method instead of block-matching methods. In this version of ASTA, the combination of nonsteering and rotation of the coordinate system is also effective, because the lateral bandwidth does not decrease. In such a case, transmission of a laterally wide wave will also be effective, particularly for three-dimensional measurement/imaging using a two-dimensional array transducer. ASTA can also be used for accurate lateral displacement measurements. Although a proper beam steering and/or a proper coordinate rotation improves the measurement accuracy achieved with LM and ASTA, all measurement accuracies obtained with ASTA are lower than with LM.
\end{abstract}

Keywords: lateral modulation, single steering angle, coordinate system rotation, spectra frequency division

\section{Introduction}

Various ultrasonic displacement/velocity measurement methods have been well developed for measurement of blood flow (eg, the continuous wave Doppler method, ${ }^{1}$ pulse wave, ${ }^{2}$ autocorrelation method, ${ }^{3}$ and cross-correlation method ${ }^{4}$ ), tissue strain (eg, Doppler method, autocorrelation method, ${ }^{5}$ cross-correlation method ${ }^{6}$ ), sonar data, and other target motions. For about 60 years, tissue axial displacement/velocity/strain has been measured using these one-dimensional methods, although other developments have allowed measurement of multidimensional displacement/velocity vectors and strain/strain rate tensors for blood flow ${ }^{7}$ (in the heart and other organs) and for tissue motion, ${ }^{8}$ eg, the multidimensional cross-correlation method.
Correspondence: Chikayoshi Sumi Department of Information and Communication Science, Faculty of Science and Technology,

Sophia University, 7-I, Kioi-cho, Chiyoda-ku, Tokyo I02-8554, Japan

$\mathrm{Tel}+81332383415$

Fax +81332383321

Email c-sumi@sophia.ac.jp
Reports in Medical Imaging 20II:4 47-66

(c) 20II Sumi et al, publisher and licensee Dove Medical Press Ltd. This is an Open Access article

Dovepress

http://dx.doi.org/10.21 147/RMI.S24568 
For these types of applications, Sumi et al have developed several displacement measurement methods. ${ }^{9-18}$ For instance, the multidimensional cross-spectrum phase gradient method, ${ }^{9,10}$ the multidimensional autocorrelation method (MAM),${ }^{11-13}$ and the multidimensional Doppler method $(\mathrm{MDM})^{11-13}$ were developed for displacement vector measurement, and specifically for simultaneous axial, lateral, and elevational displacement measurements. A displacement vector measurement method similar to MAM has also been reported by another group. ${ }^{19}$

Sumi et al have developed lateral modulation (LM) methods ${ }^{11-18}$ that are effective for displacement vector measurement. LM measurements can be made using superposition of steered crossed beams. ${ }^{12-18}$ This involves superposition of multiple steered beams, with different steering angles obtained using the multiple transmission method ${ }^{20,21}$ or synthesized from a set of received echo data using the multidirectional synthetic aperture method. ${ }^{20,21}$ Simultaneous or successive transmissions/receptions of an ultrasound signal can be used with the multiple transmission method. Multiple transducers can also be used with this type of beamforming. The LM method also permits echo imaging where the lateral resolution is almost the same as the axial resolution. ${ }^{15,16}$ In this case, the shapes of the apodization functions must also be carefully considered. ${ }^{15,16}$ For displacement vector measurement, LM can also be performed using the Fraunhofer approximation, as reported by Jensen $^{22,23}$ and Anderson. ${ }^{24,25}$ Their common approach uses an apodization function which shows several symmetric peaks. Their approach also performs analog LM processing twice to obtain multidimensional analytic signals. In contrast, the digital LM approach developed by Sumi et al requires less processing to yield the analytic signals. The displacement vectors and strain tensors that are accurately measured are also used for reconstructing the mechanical properties of, eg, the liver, heart, breast, and blood. ${ }^{26}$

Sumi et al also recently developed a beamforming method which is simpler than LM, ie, ASTA, ${ }^{17,18}$ which uses only a steering angle. All of the beamforming methods, including ASTA, can be performed on an arbitrary orthogonal coordinate system. However, LM uses two steering angles for two-dimensional measurement/imaging, and three or four steering angles for three-dimensional measurement/imaging, ie, multiple crossed beams (see Appendix A for a twodimensional example). Although LM enables measurement of various tissue displacements with arbitrary directions only by attaching an ultrasonic transducer onto the target surface, ASTA has several further advantages with respect to $\mathrm{LM}^{17,18}$ (see Appendix A).
However, with ASTA, the number of methods available to obtain a displacement vector measurement is limited to previously developed block-matching methods, ${ }^{17,18}$ such as the multidimensional cross-spectrum phase gradient method, the multidimensional cross-correlation method, and MAM and MDM using a block-matching method (referred to as $\mathrm{MAMb}$ and $\mathrm{MDMb},{ }^{17,18}$ respectively). This is because only a single quadrant or octant spectrum can be obtained. ${ }^{17,18}$ That is, MAM and MDM methods using a moving average cannot be used. In order to permit the use of MAM and MDM with ASTA for displacement vector measurement, Sumi et al proposed use of the first version of MAM and MDM without LM or beam steering, ${ }^{11,13,14,20,27}$ ie, the spectra frequency division method (SFDM), ${ }^{28-30}$ in which two-dimensional or three-dimensional analytic signals are generated by dividing the single quadrant or octant spectra in the corresponding frequency domain. As confirmed by simulations, ${ }^{17,18}$ the block-matching methods with ASTA yield a lower measurement accuracy than with LM. In this report, the accuracy of measurements obtained with SFDM and ASTA is confirmed through simulations and agar phantom experiments. ${ }^{28-30}$ For SFDM with ASTA, the effect of a manual, mechanical, or electric steering angle on measurement accuracy is also examined. Although the steering angles have already been examined for $\mathrm{LM},{ }^{13,15,16}$ in this report the effect is also evaluated using the same models as those used for the ASTA evaluation. For both LM and ASTA, the steering angles can control the frequencies of all the axes (see equation A1 in Appendix A). Briefly, it was confirmed that for both LM and ASTA, beams steered in the same direction as the target displacement vectors yield a small standard deviation (SD) for the magnitude, and a large SD for the angles.

In conjunction, we noted a new fact that these equations explain the frequency changes of all the axes generated by coordinate rotation as well as by beam steering. In this report, after specifically describing the relationship between frequency changes and the rotation angle of a coordinate system, the effect of the rotation angle on measurement accuracy is examined, mainly with LM and ASTA with SFDM using the directions of tissue motion and steering beams (steering angles) as parameters in simulations and agar phantom experiments. ${ }^{28-30}$ Rotation of the coordinate system can be realized analytically or approximately (by interpolation) when beamforming or after beamforming in a spatial or frequency domain. ${ }^{17,18}$

In addition to such displacement vector measurement methods, development of a simple lateral displacement measurement method has been an important goal. Various measurement methods have been developed, for instance the 
lateral cross-correlation method, and several methods have also been developed using ASTA by Sumi, ie, the mirror setting method, ${ }^{17,18}$ and another method which divides a multidimensional moving-averaged instantaneous phase change by a multidimensional moving-averaged lateral frequency (hereafter referred to as "lateral Doppler"). ${ }^{17,18}$ These two lateral displacement measurement methods yield a high measurement accuracy in a simpler manner than the conventional Doppler method with use of the rotational angle of a transducer when used with ASTA, ${ }^{17,18}$ and with multidimensional measurement methods used with LM. For instance, these two methods can be used for measuring blood flow in vessels running parallel to the body surface (eg, the carotid artery). However, for the two lateral measurement methods, the lateral coordinate axis must be set in the direction of the target lateral motion by rotating the coordinate system analytically or approximately (by interpolation) when beamforming or after beamforming, in a similar manner. ${ }^{17,18}$ In this report, the effect of coordinate rotation (interpolation error) in addition to the steering angle on lateral measurement accuracy is also examined using simulations and agar phantom experiments.

\section{Displacement vector measurements Spectra frequency division method}

Figure 1 shows a two-dimensional displacement vector measurement, ie, a schematic of the spectra frequency division for SFDM (Figure 1B) with ASTA $\theta$ (Figure 1A). Such a single quadrant spectra is divided to obtain two spectra for MAM and MDM, eg, by using a linear line passing through the center of the spectra and a direct current. The first moment of the spectra can be used as the center of the spectra. That is, the following simultaneous equations for axial $(\mathrm{dx})$ and lateral (dy) displacements are obtained from the two spectra (ie, the two synthesized beams 1 and 2 shown in Figure 1B):

$$
f_{x 1} d x+f_{y 1} d y=c_{1} \text { and } f_{x 2} d x+f_{y 2} d y=c_{2}
$$

for which coefficients are synthesized by the spectra division, $\mathrm{ie}$, the instantaneous phase changes $\mathrm{c}_{1}$ and $\mathrm{c}_{2}$, and axial $\left(\mathrm{f}_{\mathrm{x} 1}\right.$ and $\mathrm{f}_{\mathrm{x} 2}$ ) and lateral instantaneous $\left(\mathrm{f}_{\mathrm{y} 1}\right.$ and $\mathrm{f}_{\mathrm{y} 2}$ ) frequencies.

With the first version of MAM and MDM, ${ }^{11,13,14,20,27}$ neither LM nor any beam steering was used. That is, $\mathrm{SFDM}^{28-30}$ was used only for nonsteered beams with $\theta=0^{\circ}$. In the simulations and agar phantom experiments, the nonsteered beams were also used as a version of ASTA, for comparison purposes.

As described later, in conjunction with a steering angle, the proper direction of the division must be considered. A curved line can also be used instead of a linear line. The
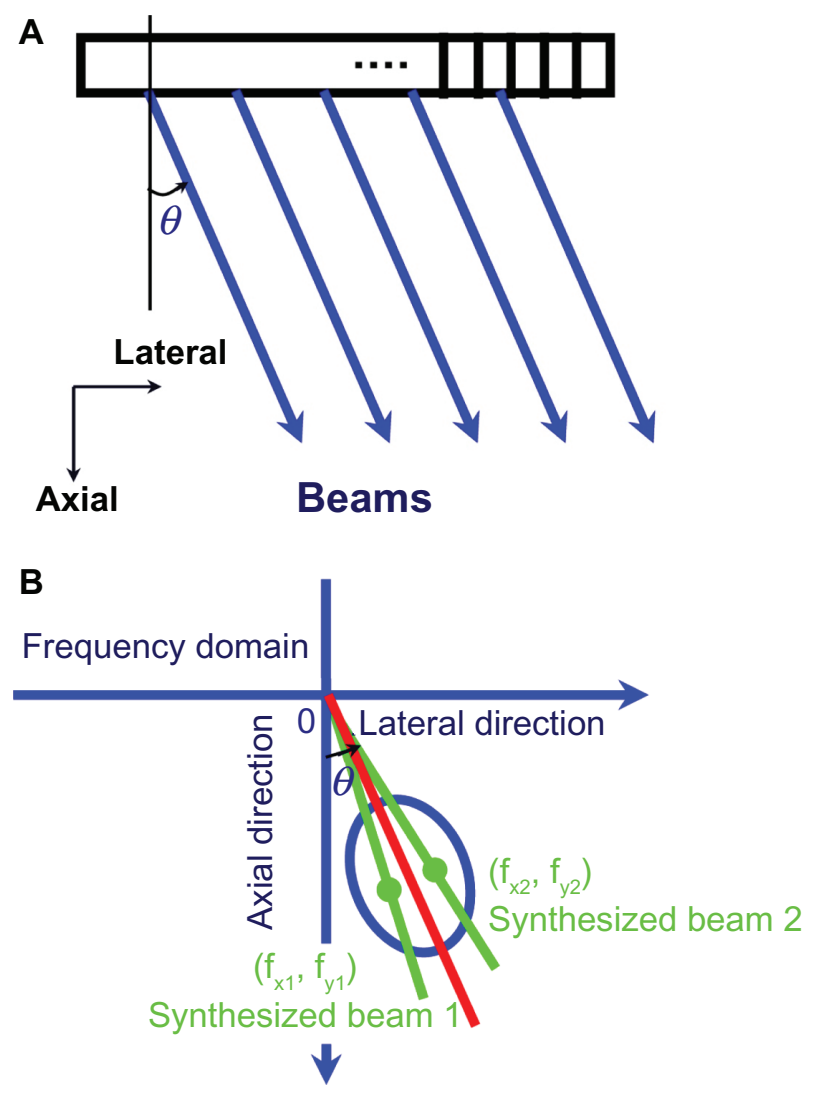

Figure I (A) With a steering angle (ASTA) $\theta$, (B) schematic of the spectra frequency division method. For two-dimensional displacement vector measurements, for instance, a single quadrant spectra is divided to obtain two spectra for the multidimensional autocorrelation method and the multidimensional Doppler method using a linear line passing through a direct current. Beams $I$ and 2 are synthesized and, correspondingly, the first moments $\left(f_{x 1}, f_{y 1}\right)$ and $\left(f_{x 2}, f_{y 2}\right)$ are obtained.

lines do not always have to pass through the direct current and the center of the spectra. A number of spectra larger than the two spectra obtained using plural lines can also be used, in which case the over determined simultaneous equations obtained are solved for the displacement vector components using the least squares method, or the plural measurement results calculated using differnt combinations of divided spectra are superposed (ie, averaged). The spectra division can also be performed by weighting the spectra using windows. The divided spectra may partially include the same spectra. Original spectra may also be used. Further, the spectra may be partially disregarded, eg, low frequency spectra may be disregarded to increase instantaneous frequencies. ${ }^{28-30}$ Spectra bordering the division boundaries may also be disregarded to increase the independence of simultaneous equations. For three-dimensional displacement vector measurement, a single octant spectra (eg, Figure 2B from reference 18) for a three-dimensional case is divided using more than two linear or curved planes similarly. Correspondingly, at least three independent equations are derived. The SFDM can further be 
used for the LM case (see Figure 2C from reference 18 for a two-dimensional example).

\section{Simulations}

First, simple simulations were performed to examine the effect on measurement accuracy obtained with ASTA, ie, a manual, mechanical, or electric steering angle. For two-dimensional displacement vector measurement, the same rigid lateral motion $\left(\theta=90^{\circ}\right)$ was used as described elsewhere by Sumi, ${ }^{18}$ ie, $0.01 \mathrm{~mm}$. Echo data were simulated by convolving white noise data in a two-dimensional region of interest with a Gaussian-type point spread function and a
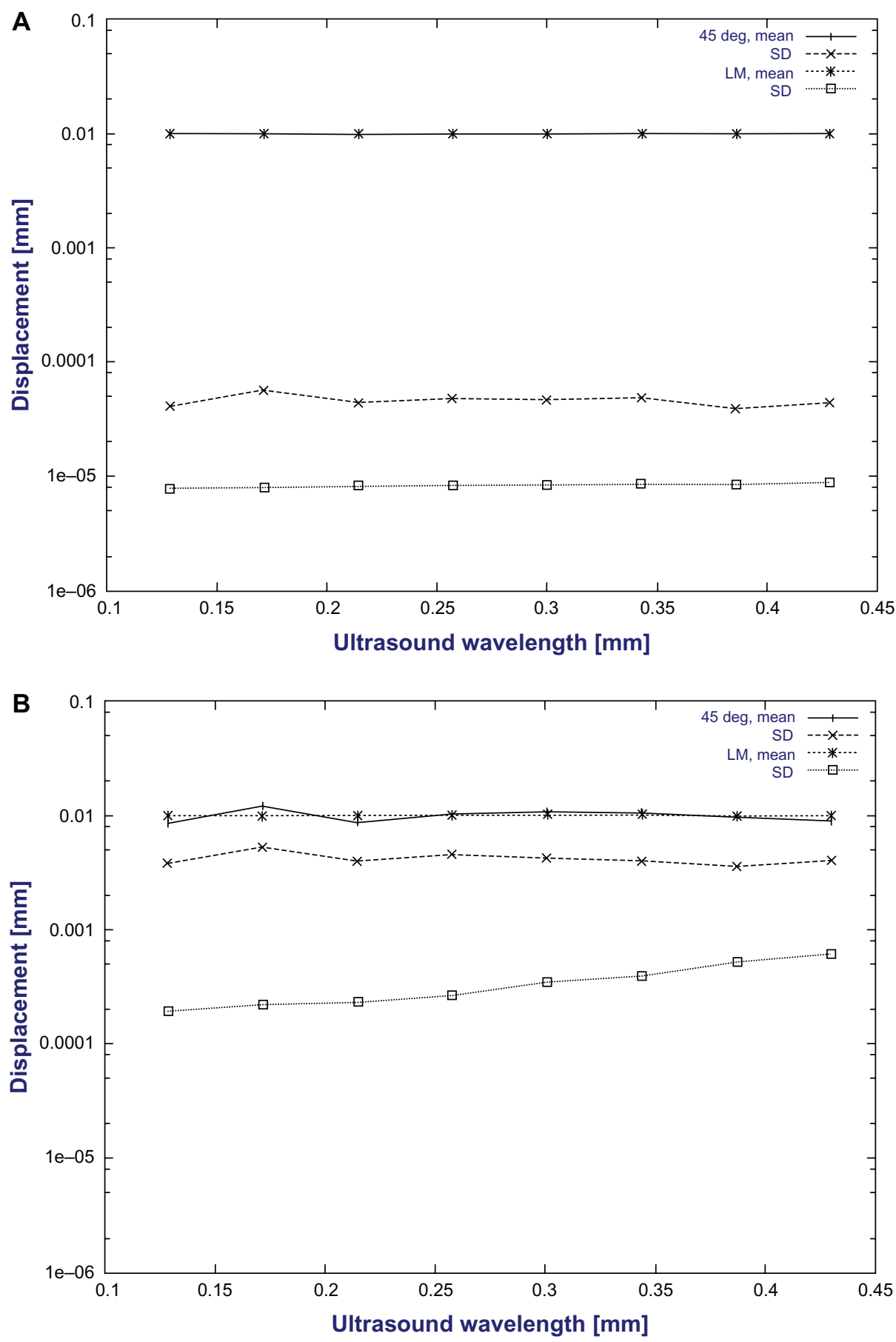

Figure 2 For simulated echo data with lateral displacement (magnitude, $0.01 \mathrm{~mm}$; and $\theta=90^{\circ}$ in Figure IA), evaluation of the statistics (means and standard deviations [SDs]) of lateral displacement measured using two-dimensional autocorrelation method with spectra frequency division method and ASTA for (A) no noise and (B) for $20 \mathrm{~dB}$ echo signal-to-noise ratio cases. Values for lateral modulation can also be compared.

Abbreviations: deg, degrees; SD, standard deviation; LM, lateral modulation; mm, millimeters. 
spatially isotropic SD of $0.4 \mathrm{~mm}$. The ultrasonic frequency was changed from 3.5 to $12.0 \mathrm{MHz}$ for a two-dimensional medium with a ultrasonic speed of $1500 \mathrm{~m} / \mathrm{sec}$, and the steering angle $\theta$ was set at $45^{\circ}$ (see $\theta$ in Figure 1A). An axial sampling frequency of $30 \mathrm{MHz}$ and a beam pitch of $0.05 \mathrm{~mm}$ were used. Considering the Nyquist theorem, if necessary, the lateral bandwidth was increased using the method described previously by the author ${ }^{18}$ as shown in Appendix C. By adding white noise to the raw echo data, echo data with a signal-to-noise ratio of $20 \mathrm{~dB}$ were also simulated. Here LM with SFDM was not simulated, but a pure LM case was also simulated for two-dimensional displacement vector measurement, in which steering angles of $\pm 45^{\circ}$ were used. The two-dimensional autocorrelation method was used for all the displacement vector measurements.

Figure 2 shows the ultrasonic frequency vs the means and standard deviations (SDs) of the lateral displacements measured using the two-dimensional autocorrelation method with LM and with SFDM and ASTA for two cases, ie, no noise and a $20 \mathrm{~dB}$ echo signal-to-noise ratio. In this simulation, the axial and lateral frequencies were the same, thus for respective displacement vector measurement, the SDs of the axial and lateral displacement measurements were almost the same under zero axial displacement (axial data omitted).

As shown, unbiased measurements were performed (see the evaluated means). When using other displacement vector measurement methods, such as the two-dimensional crossspectrum phase gradient method and the two-dimensional Doppler method, similar results were obtained (data omitted). The corresponding results obtained using the twodimensional multidimensional cross-spectrum phase gradient method, two-dimensional $\mathrm{AMb}$ (ie, a two-dimensional autocorrelation method with block-matching), and twodimensional DMb (ie, a two-dimensional Doppler method with block-matching) are shown in a previous publication by Sumi. ${ }^{18}$ When performing such block-matching, ASTA resulted in a SD about 100 times as large as with pure LM, and when using SFDM, ASTA resulted in a SD about ten times as large as that for the pure LM method.

Figure 3 shows, for various directions of a displacement vector $\left(\theta=0^{\circ}-90^{\circ}\right.$, Figure $\left.1 \mathrm{~A}\right)$, the steering angle $\theta=0^{\circ}-90^{\circ}$ vs the SDs of the magnitude and angle of the respective displacement vectors (magnitude $0.01 \mathrm{~mm}$ ) measured using the two-dimensional autocorrelation method with LM (Figure 3A), and with SFDM and ASTA (Figure 3B). LM was achieved by generating steering angles of $-\theta$ as well. The ultrasonic frequency used was $7 \mathrm{MHz}$. This also confirmed that LM yields more accurate measurements than ASTA (SFDM) (see Figure 3A vs B). For beamforming in both cases, when the SD of the magnitude is large, that of the angle is small, and vice versa. It was also confirmed that proper steering angles exist for the respective directions of the displacement vectors. For both LM and ASTA, beams steered in the same direction as the target displacement vectors yielded a small SD for the magnitude and a large SD for the angles. In addition, the $\theta$ steering angles should be set at $45^{\circ}$ to deal simultaneously with the various directions of the displacement vectors.

Figure $3 \mathrm{C}$ shows the SDs obtained for LM using steering angles of $\pm 10^{\circ}$ and $\pm 20^{\circ}$ when changing the angle at the center of the two steered beams. As shown, if the central angles are the same as those of the target displacement vectors, there are small SDs for the magnitude and large SDs for the angles. It should also be noted that the use of symmetric beams with respect to the axial direction yields accurate measurements, particularly when beam steering is performed at $\pm 20^{\circ}$ (compare the angle at the center of the two beams, $0^{\circ}$ vs $\left.10^{\circ}\right)$.

\section{Agar phantom experiments}

Next, for an agar phantom, ASTA and LM were performed using a linear array transducer. For ASTA, SFDM was used. The agar phantom used was the same as that described elsewhere by Sumi et al. ${ }^{15}$ The rectangular region of interest with 13.7 (axial) $\times 13.2$ (lateral) $\mathrm{mm}$ dimensions was set at a depth of $12.2 \mathrm{~mm}$, in which a central circular region (a cylindrical inclusion with a radius of $10 \mathrm{~mm}$ and a depth of $19 \mathrm{~mm}$ ) had a larger shear modulus than the surrounding region, ie, a relative value of $3.29\left(2.63\right.$ vs $\left.0.80 \times 10^{6} \mathrm{~N} / \mathrm{m}^{2}\right)$. The agar phantom was compressed in the lateral direction. The ultrasonic frequency used was 7.5 MHz. Parabolic functions were used for apodizations of ASTA and LM. ${ }^{15,16}$ Steering angles $\theta= \pm 11.9^{\circ}$ were used.

In Figure 4A, for SFDM, a single quadrant spectra was divided by four straight lines (i-iv) passing through the first moment of the spectra. The single quadrant spectra is shown in Figure 4B (first on left). The statistics of the magnitude and angle of the displacement vectors measured in a central circular region (radius $=2.5 \mathrm{~mm}$ ) of the inclusion were evaluated (see Figure 4C). The results for $\theta$ minus $90^{\circ}$ are shown (the mean is nearly $7.6^{\circ}$ ). The lateral compression which was performed was slanted by $7 \cdot 6^{\circ}$. As shown, for the small steering angle $\left(11.9^{\circ}\right)$, a vertical division, such as the one made using lines i and iii, should be performed rather than a horizontal division, such as one made using lines ii and 

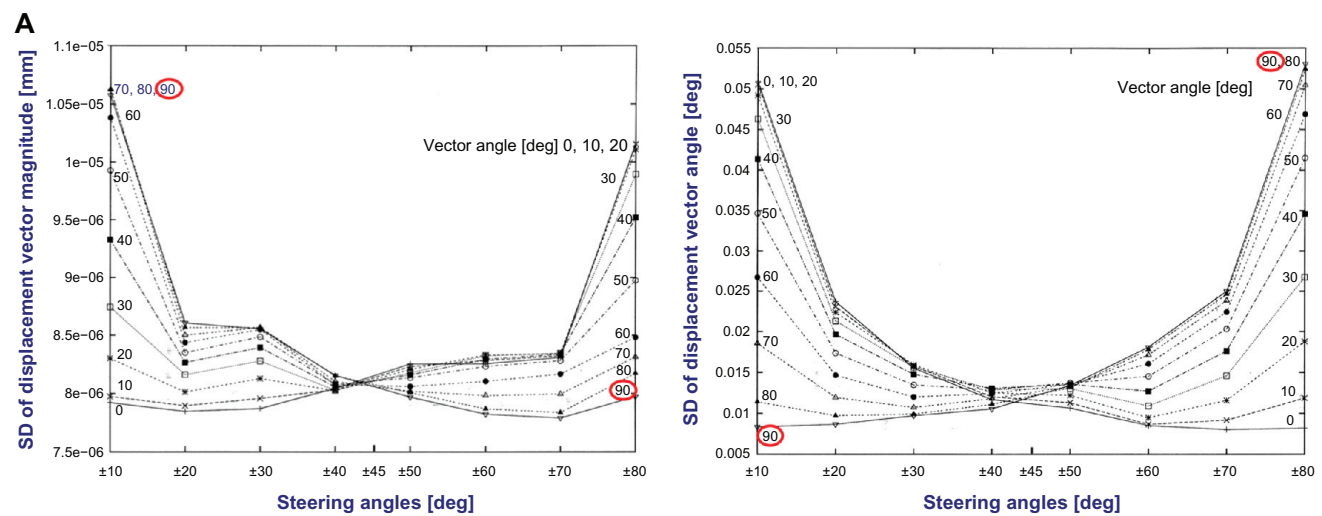

\section{B}
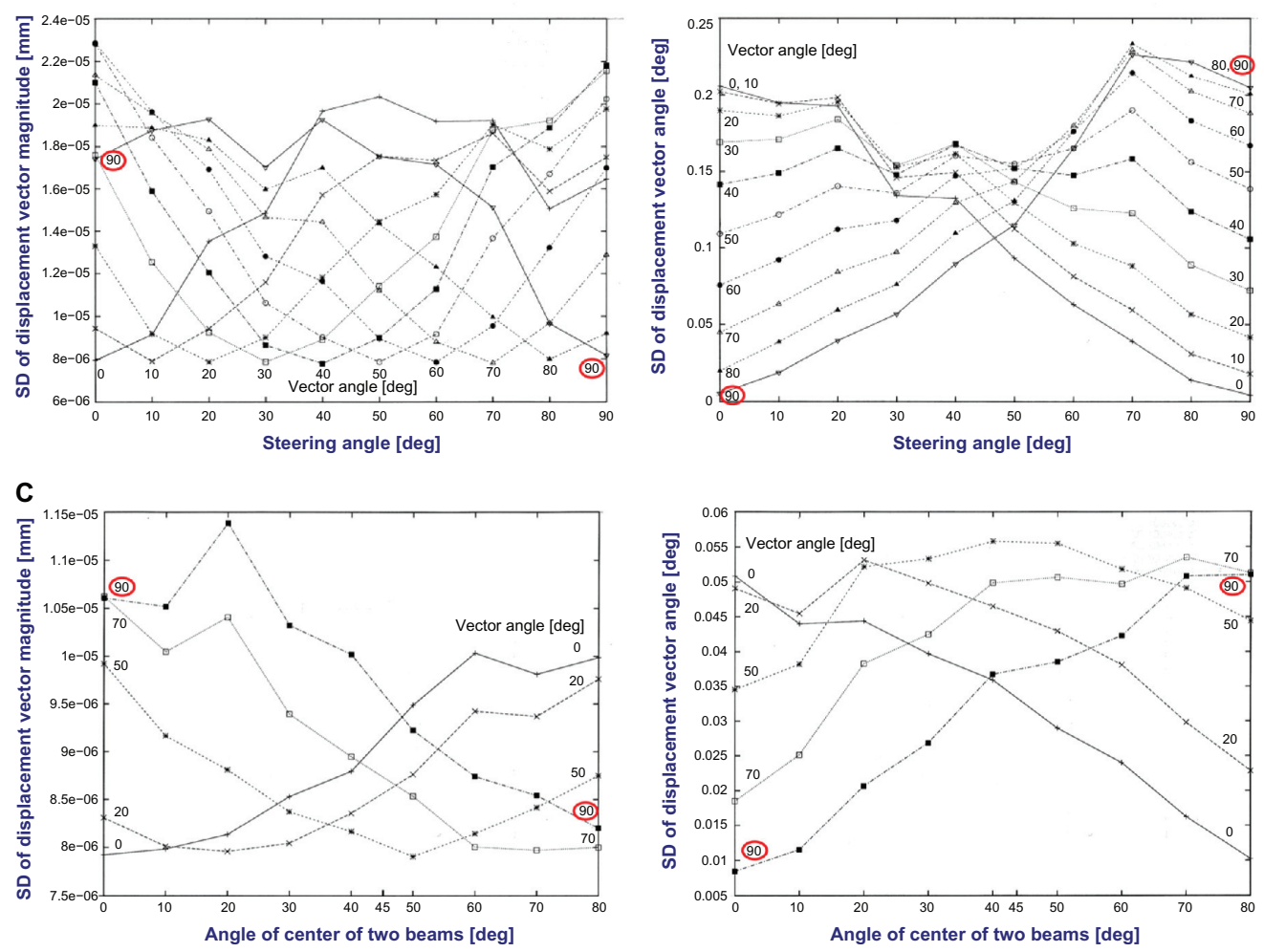

Steering angles, $\pm 10[\mathrm{deg}]$
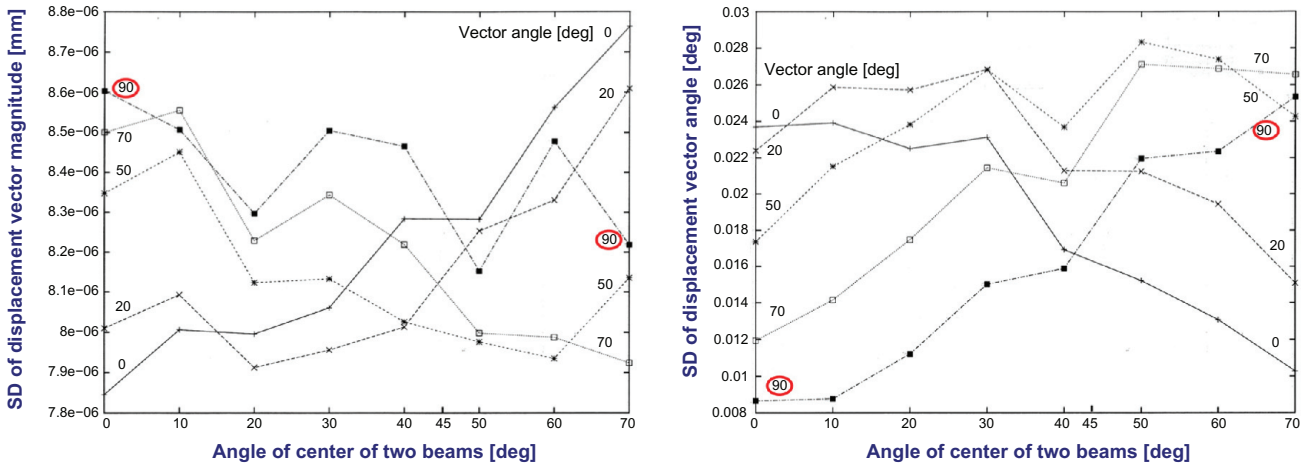

Steering angles, $\pm 20[\mathrm{deg}]$

Figure 3 For the same simulated echo data with lateral displacement (magnitude, $0.01 \mathrm{~mm} ; \theta=90^{\circ}$ in Figure IA), evaluation of standard deviations of the displacement vector (magnitude and angle) measured (A) using two-dimensional autocorrelation method with lateral modulation and (B) using spectra frequency division method with ASTA vs steering angles. (C) For lateral modulation with steering angles of $\pm 10^{\circ}$ and $\pm 20^{\circ}$, SDs vs the angle at the center of the two steered beams.

Abbreviations: $\mathrm{mm}$, millimeters; deg, degrees; $\mathrm{SD}$, standard deviation. 
A

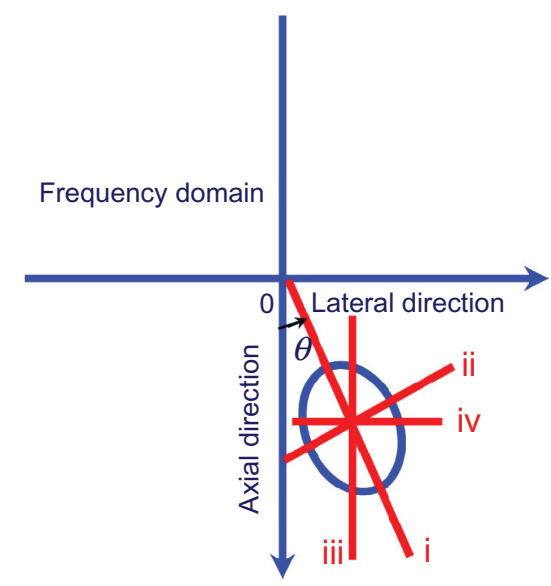

B

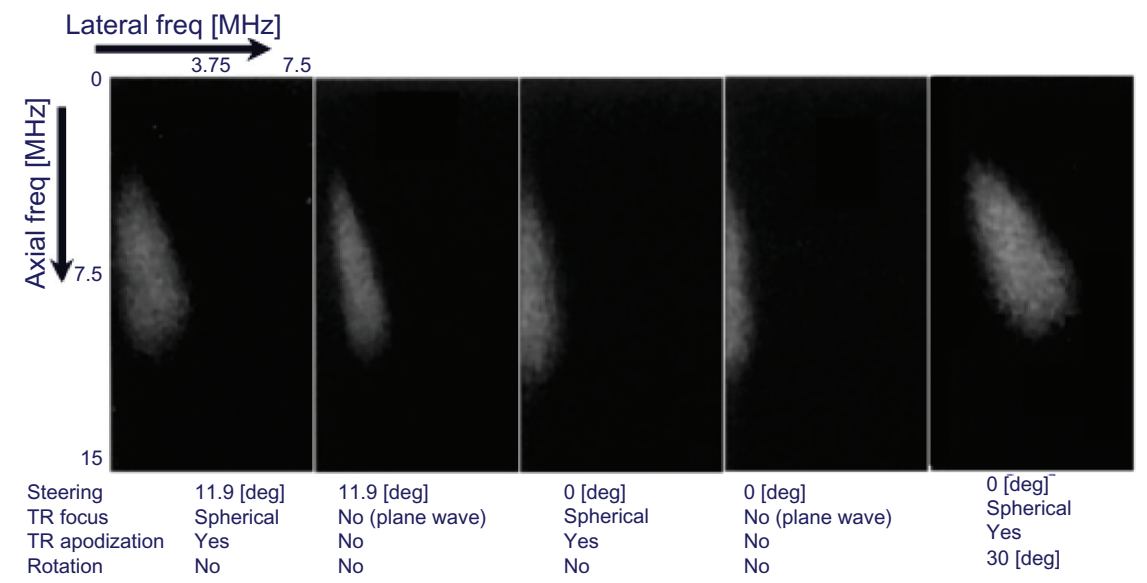

C
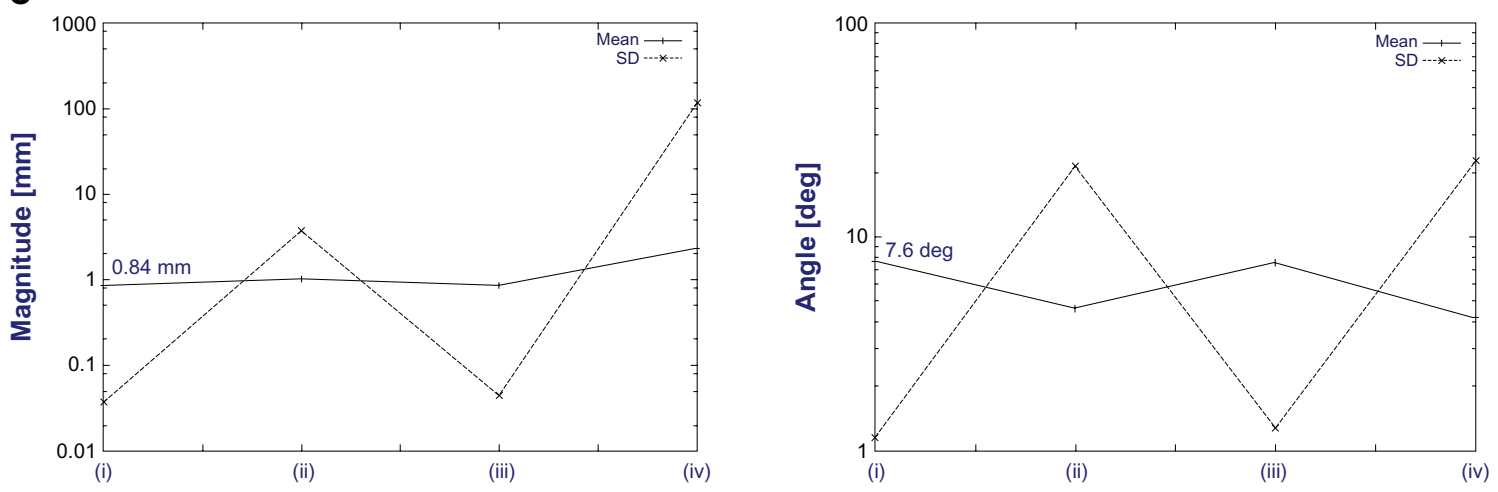

Figure 4 (A) Examples of a spectra frequency division (schematic) i-iv. (B) Spectra obtained for an agar phantom. ${ }^{15,16}$ (C) For an agar phantom compressed in a lateral direction $\theta=90^{\circ}$ (see Figures $4 \mathrm{~A}$ and IA), the direction of divisions $\mathrm{i}$-iv vs the statistics of the measured displacement vectors in a central circular region (radius $=2.5 \mathrm{~mm}$ ) in a stiff inclusion, ie, those of the magnitude and measured angle of $\theta$ minus $90^{\circ}$.

Abbreviations: $\mathrm{MHz}$, megahertz; deg, degrees; TR, transmission; SD, standard deviation.

iv. For a steering angle larger than $45^{\circ}$, a horizontal division should be performed (data not shown).

\section{Rotation of coordinate system}

A rotation of the coordinate system was next performed (see Figure 5). As mentioned in the Introduction, equation A1 in Appendix A can be applied to the steering angle $\theta$ minus $\theta^{\prime}$, defined on the new coordinate system (depicted by red) and rotated by angle $\theta^{\prime}$ instead of the steering angle $\theta$ defined on the old coordinate system (black) as follows:

$$
\mathrm{f}_{\mathrm{x}}^{\prime}=\mathrm{f}_{0} \cos \left(\theta-\theta^{\prime}\right) \text { and } \mathrm{f}_{\mathrm{y}}^{\prime}=\mathrm{f}_{0} \sin \left(\theta-\theta^{\prime}\right),
$$

where $\mathrm{f}_{0}, \mathrm{f}_{\mathrm{x}}{ }^{\prime}$, and $\mathrm{f}_{\mathrm{y}}^{\prime}$ are, respectively, ultrasonic frequency and axial $\left(\mathrm{x}^{\prime}\right)$ and lateral $\left(\mathrm{y}^{\prime}\right)$ frequencies of the new coordinate system. The frequencies $\mathrm{f}_{\mathrm{x}}{ }^{\prime}$ and $\mathrm{f}_{\mathrm{y}}{ }^{\prime}$ are expressed using 


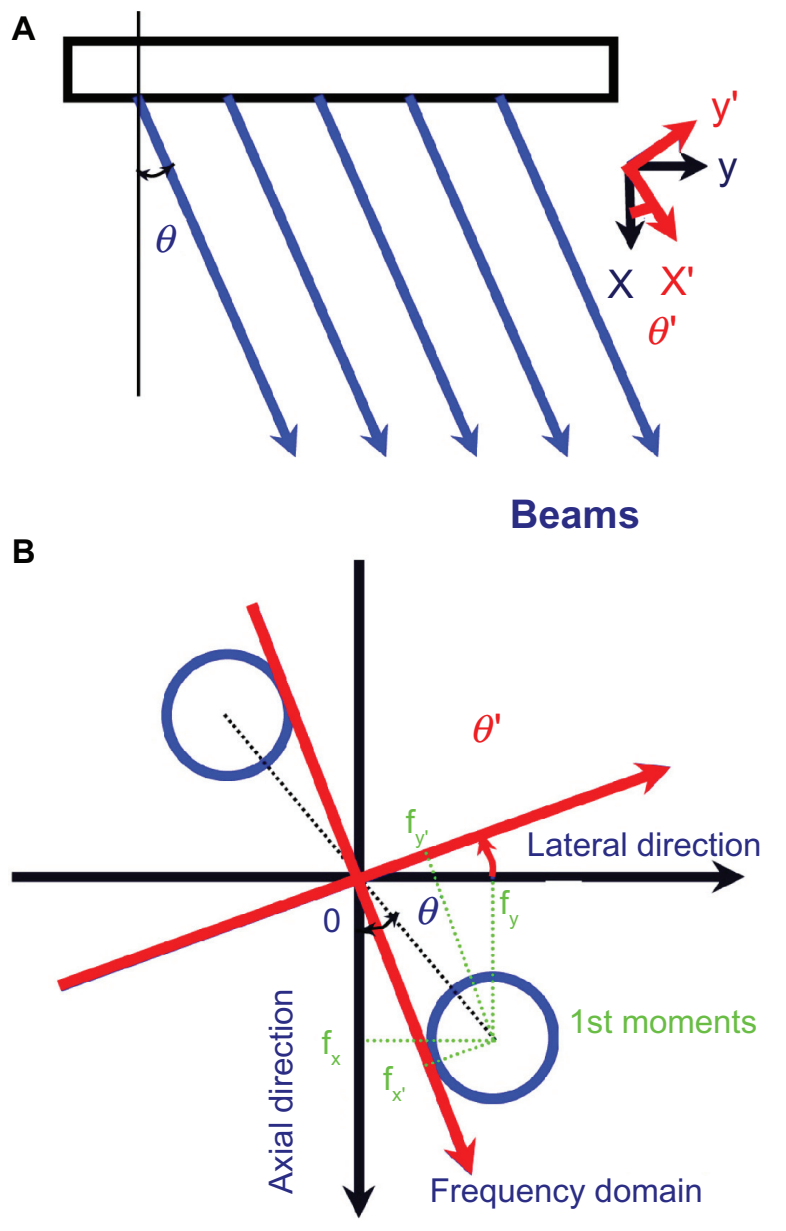

Figure 5 Rotation of coordinates in (A) spatial and (B) frequency domains by $\theta^{\prime}$ (schematic drawing in red). In Figure 5B, the first moments of the spectra are shown as $\left(f_{x}, f_{y}\right)$ and $\left(f_{x}^{\prime}, f_{y}{ }^{\prime}\right)$ on the old and new coordinates, respectively.

fx and fy of the old coordinate system (see equation A1) and a rotation matrix as follows

$$
\left(\begin{array}{l}
f_{x}^{\prime} \\
f_{y}^{\prime}
\end{array}\right)=\left(\begin{array}{cc}
\cos (-\dot{\theta}) & -\sin (-\dot{\theta}) \\
\sin (-\dot{\theta}) & \cos (-\dot{\theta})
\end{array}\right)\left(\begin{array}{l}
f_{x} \\
f_{y}
\end{array}\right) .
$$

If a laterally symmetric beam with the same ultrasonic frequency, $\mathrm{f}_{0}$, is also generated and crossed at position ( $\left.\mathrm{x}, \mathrm{y}\right)$ for LM (see Appendix A), simultaneous equations of MAM or MDM about the unknown displacement vector ( $\mathrm{dx}$, dy) of the position are expressed on the old coordinate system: ${ }^{11-13}$

$$
f_{x} d x+f_{y} d y=c_{1} \text { and } f_{x} d x-f_{y} d y=c_{2},
$$

where $c_{1}$ and $c_{2}$ are instantaneous phase changes in the two steered beam directions.

On the new coordinate system, the first part of equation (4) is expressed as:

$$
f_{x}^{\prime} d x^{\prime}+f_{y}^{\prime} d y^{\prime}=c_{1}
$$

where $\mathrm{dx}^{\prime}$ and $\mathrm{dy}^{\prime}$ are unknown displacement vector components of the position $(x, y)$ expressed on the new coordinate system, and have the following relationship with (dx, dy) and the same rotation matrix as that used in equation (3).

$$
\left(\begin{array}{l}
\mathrm{d}_{\mathrm{x}}^{\prime} \\
\mathrm{d}_{\mathrm{y}}^{\prime}
\end{array}\right)=\left(\begin{array}{cc}
\cos (-\dot{\theta}) & -\sin (-\dot{\theta}) \\
\sin (-\dot{\theta}) & \cos (-\dot{\theta})
\end{array}\right)\left(\begin{array}{l}
\mathrm{d}_{\mathrm{x}} \\
\mathrm{d}_{\mathrm{y}}
\end{array}\right) .
$$

That is, the first part of equation (4) is the same as equation (5). The corresponding second part of equation (4) can be derived similarly. Also, for SFDM, the same simultaneous equations can be derived on the old equation (1) and new coordinate systems similarly.

However, we need to note that such simultaneous equations obtained on the old and new coordinate systems will not yield the same displacement vector measurement. As shown by equations (3)-(5), the respective errors in $\mathrm{f}_{\mathrm{x}}, \mathrm{f}_{\mathrm{y}}, \mathrm{c}_{1}$, and $\mathrm{c}_{2}$ due to calculations or echo noise propagate to the measurements $d_{x}{ }^{\prime}$ and $d_{y}{ }^{\prime}$, being dependent on the rotation angle $\theta^{\prime}$. Both measurement errors in magnitude and angles of displacement vectors, $(\mathrm{dx}, \mathrm{dy})$ and $\left(\mathrm{dx}^{\prime}, \mathrm{dy}^{\prime}\right)$, do not become the same.

\section{Simulations}

Using the same simulated phantoms (displacement magnitude $0.01 \mathrm{~mm}$, angles $\theta=0^{\circ}-90^{\circ}$ ), the rotation angle $\theta^{\prime}$ vs the SDs of the magnitudes and angles of the measured displacement vectors were evaluated. Figure $6 \mathrm{~A}$ and $\mathrm{B}$, respectively, show the results obtained for LM (steering angles $\theta= \pm 10^{\circ}$ and $\pm 20^{\circ}$ ) and $\operatorname{SFDM}$ (no steering, ie, $\theta=0^{\circ}$ ). The corresponding means were accurate (omitted). As shown, depending on the direction of the target displacement vector, the measurement accuracy differs. In order to deal with various directions of the displacements using LM with the steering angles, the rotation angles should be respectively smaller than $10^{\circ}$ and $20^{\circ}$. Figure $6 \mathrm{~A}$ also shows that if the beams generated with respect to the axial direction $\left(0^{\circ}\right)$ are not symmetric, a coordinate rotation should be performed such that the steered beams are symmetric. Alternatively, for SFDM, the rotation angle should be set at about $30^{\circ}$ (Figure 6B).

\section{Agar phantom experiments}

The same agar phantom echo data obtained from the synthetic aperture in the previous section were used. The displacement vector generated corresponds to $\theta=90^{\circ}$ in the simulations shown. For $\operatorname{LM}\left(\theta= \pm 11.9^{\circ}\right)$, ASTA $\left(\theta=0^{\circ}\right.$ and $11.9^{\circ}$, respectively), and the rotation $\left(\theta^{\prime}=11.9^{\circ}\right.$ and $\left.25.9^{\circ}\right)$ with ASTA $\left(\theta=0^{\circ}\right)$, Figures 7 and 8 show the statistics evaluated for displacement vectors (Figure 7A), strain tensors (Figure 7B), 
A

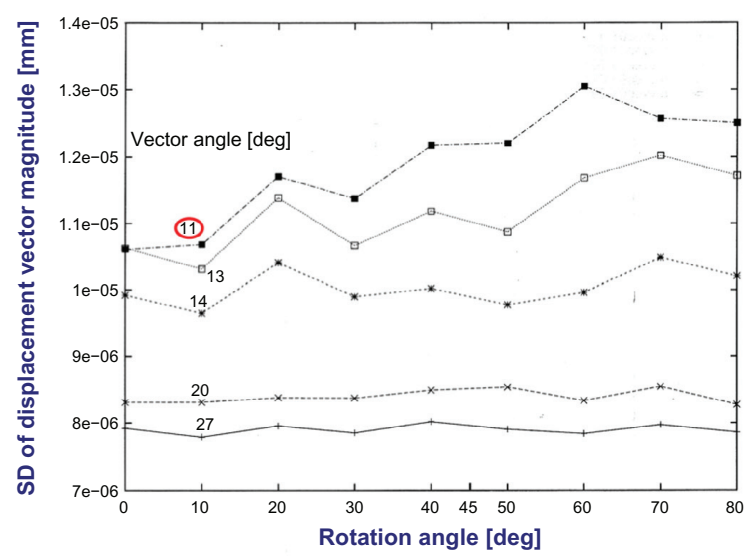

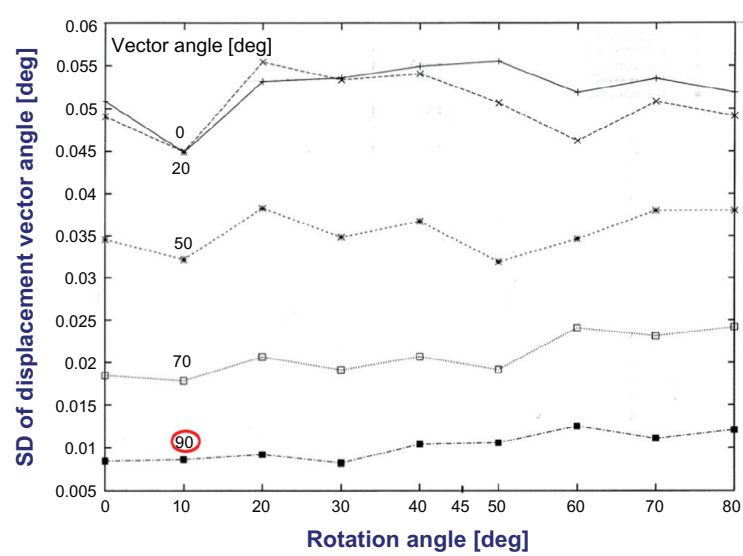

Steering angles, \pm 10 [deg]
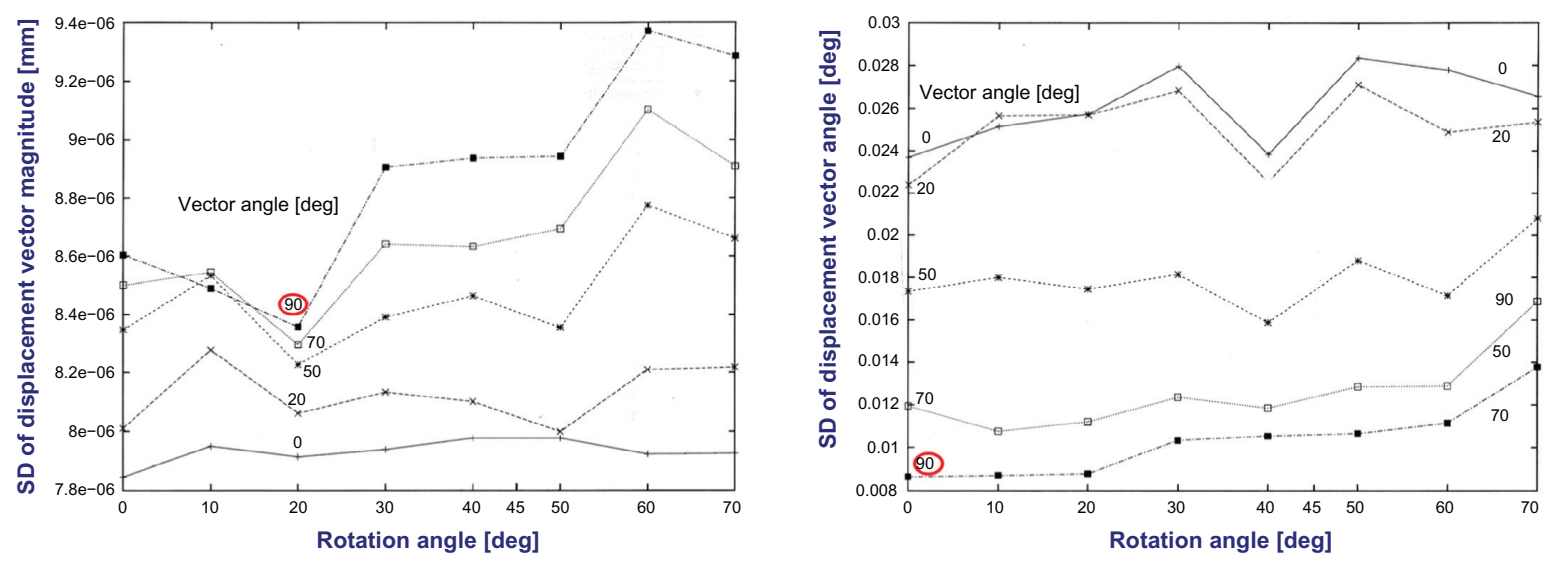

B

Steering angles, \pm 20 [deg]
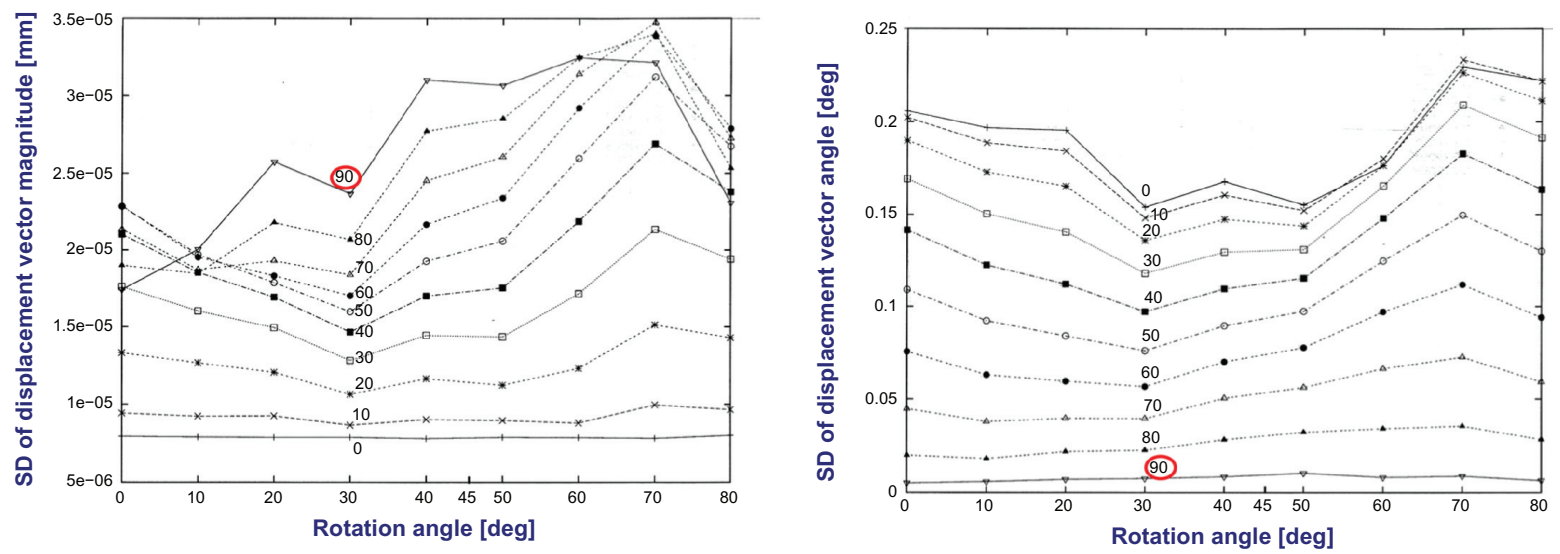

Figure 6 For the same simulated echo data with displacements (magnitude, $0.01 \mathrm{~mm}$ ) in various directions ( $\theta$ in Figure IA), evaluation of standard deviations of the magnitude and angle of displacement vector measured using the two-dimensional autocorrelation method for $(\mathbf{A})$ lateral modulation with steering angles $\theta= \pm 10^{\circ}$ and $\pm 20^{\circ}$, and (B) spectra frequency division method and ASTA vs rotation angle $\theta^{\prime}$ (see Figure 5).

Abbreviations: $\mathrm{mm}$, millimeters; deg, degrees; $\mathrm{SD}$, standard deviation.

and shear moduli (Figure 7C), measured in the central circular region (displacement vector, radius $=2.5 \mathrm{~mm}$; others, $3.2 \mathrm{~mm}$ ), and the corresponding gray-scaled images (sizes of regions of interest). Strictly, the nonsteered beam (ie, $\theta=0^{\circ}$ ) was dealt with as a version of ASTA, ${ }^{28-30}$ ie, the first version of MAM and MDM without LM. ${ }^{11,13,14,20,27}$ Because such a beam yields a larger lateral bandwidth than a steered beam, SFDM becomes more effective, as will be shown next.

In addition, a plane wave was also used for transmission, with considerations for its application to high speed scanning, ${ }^{15,16,28-30} \mathrm{eg}$, for rapid blood flow, heart, muscle, and liver motion, and three-dimensional imaging/measurement 

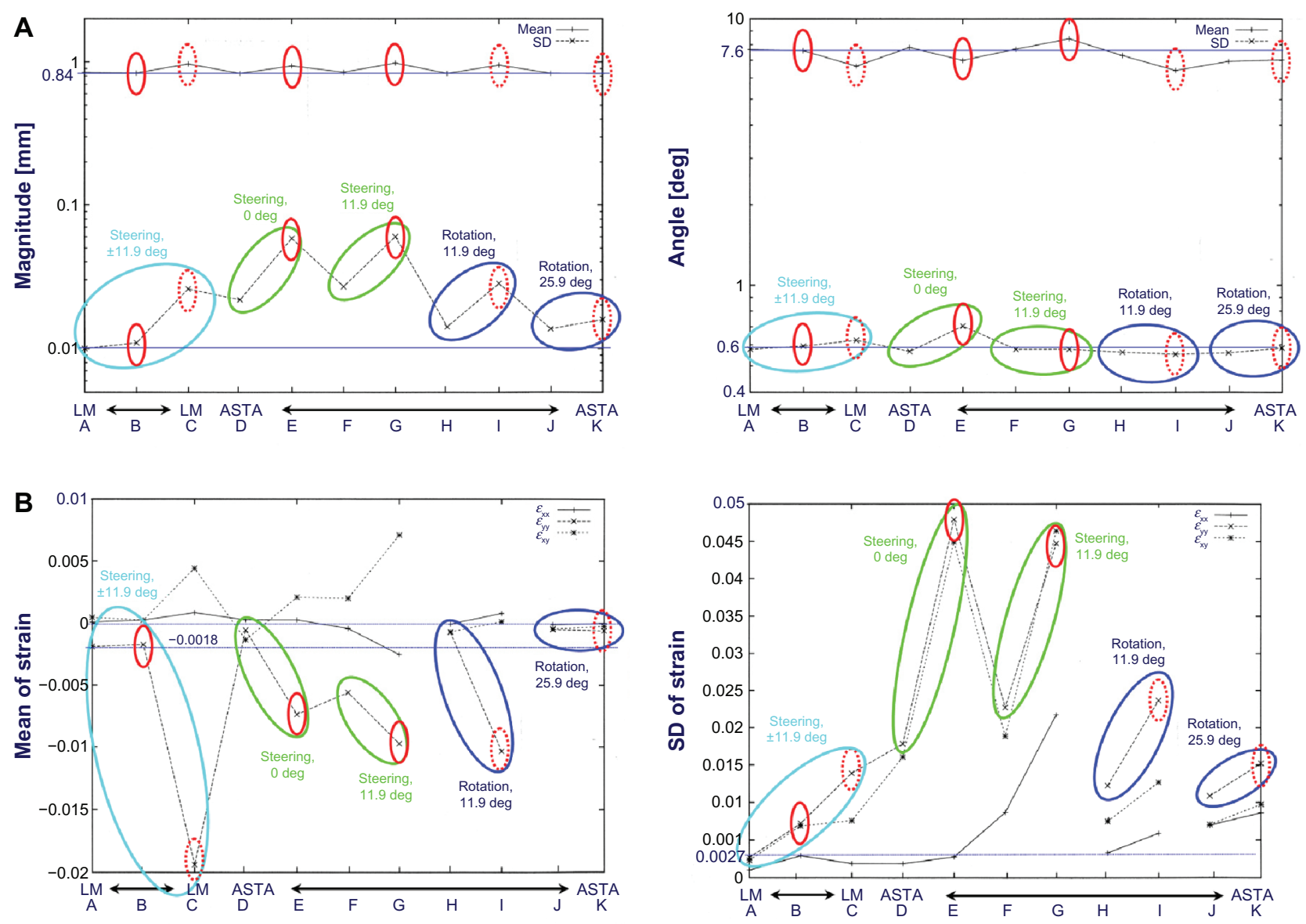

C

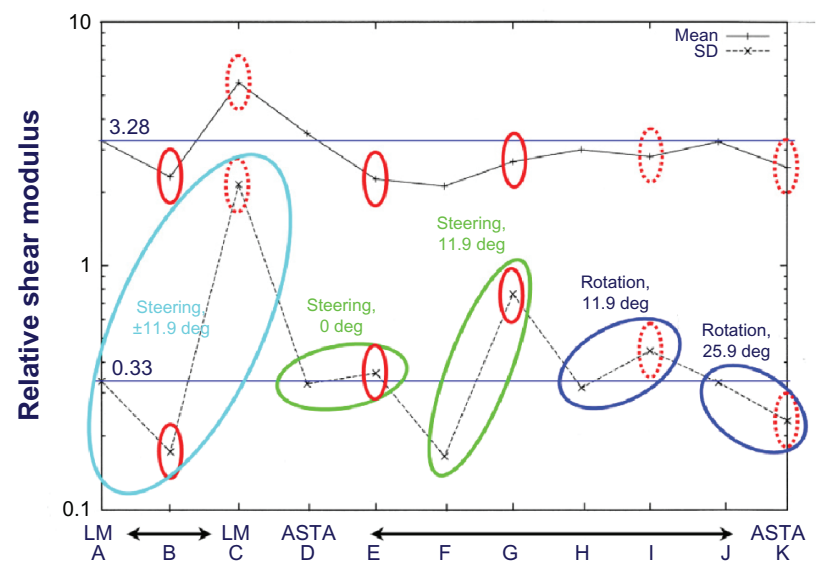

Figure 7 For the same agar phantom compressed in a lateral direction $\left(\theta=0^{\circ}\right.$ in Figure IA), evaluation of statistics for the (A) displacement vector, (B) strain tensor (axial, lateral and shear), and (C) shear modulus measured in the stiff inclusion (displacement vector, in a circular region with a radius = $2.5 \mathrm{~mm}$; others, $3.2 \mathrm{~mm}$ ). For the transmission of ultrasound, both red circles and red dot circles use plane waves, whereas noncircled areas use spherical focusing. Specifically, the red circles and red dot circles respectively use plane waves with the same steering angles as those for reception and with no steering. For strains, only lateral strains are circled.

Note: Corresponding gray-scale images are shown in Figures 8A-K.

Abbreviations: mm, millimeters; deg, degrees; LM, lateral modulation; SD, standard deviation; ASTA, a steering angle.

using a two-dimensional ultrasonic array transducer. Specifically, both the red circles and the red dot circles show the results obtained using plane waves for transmission, whereas noncircled areas show the results obtained with spherical focusing. Specifically, for the circles, the red circles use plane waves with the same steering angles as those for reception, ${ }^{15,16}$ whereas the red dot circles do not use steering. ${ }^{15,16}$ For strains, the results of the dominant lateral strain are circled (ie, Figure 7B).

Figure 4B also shows spectra obtained for steering angles of $0^{\circ}$ (third panel from the left); for a low lateral modulation frequency generated by a nonsteered plane wave transmission 
(fourth panel from the left); for $11.9^{\circ}$ (leftmost panel); for a smaller lateral bandwidth generated by a steered plane wave transmission (second panel from the left); and for a rotation angle of $30^{\circ}$ (rightmost panel).

For the displacement vector measurements obtained from such beamforming, almost the same mean values are obtained (Figure 7A). However, when using plane waves for nonfocusing transmission (red or red dot circles), except for LM using steered plane waves (a red circle, Figure 8B) and ASTA using a rotation of $25.9^{\circ}$ (Figure $8 \mathrm{~K}$ ), the SDs obtained are larger than those obtained using the corresponding spherical focusing, because the lateral bandwidths obtained are smaller. Also, compare the corresponding images in Figures 8A-K (eg, Figure 8A vs C). When using
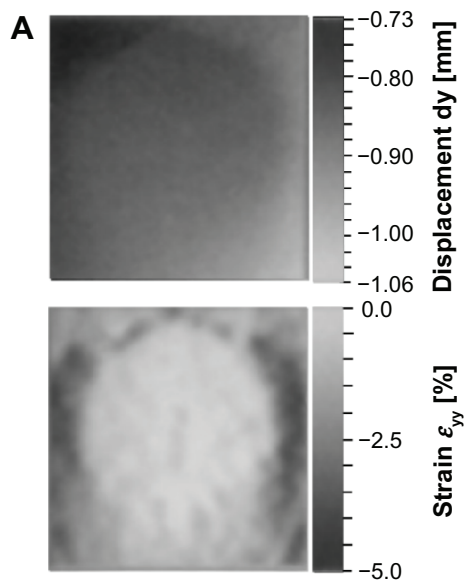

B
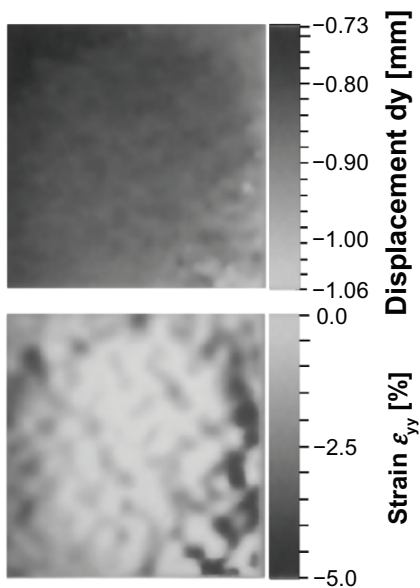

C
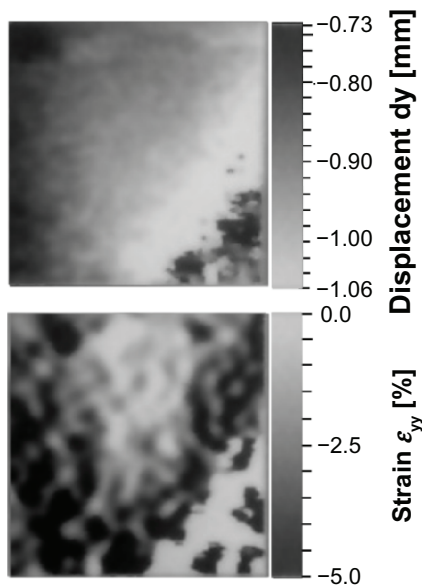
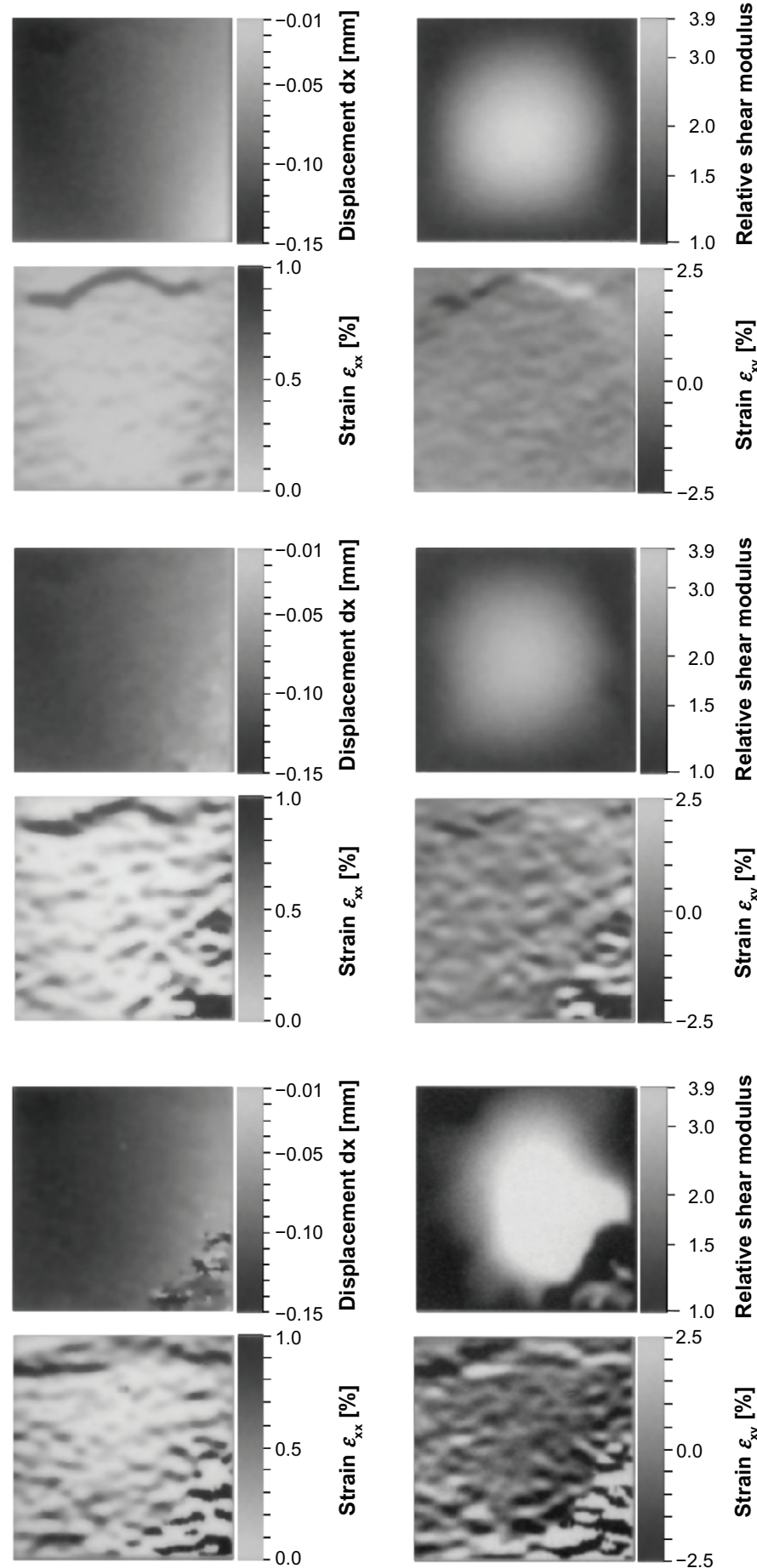
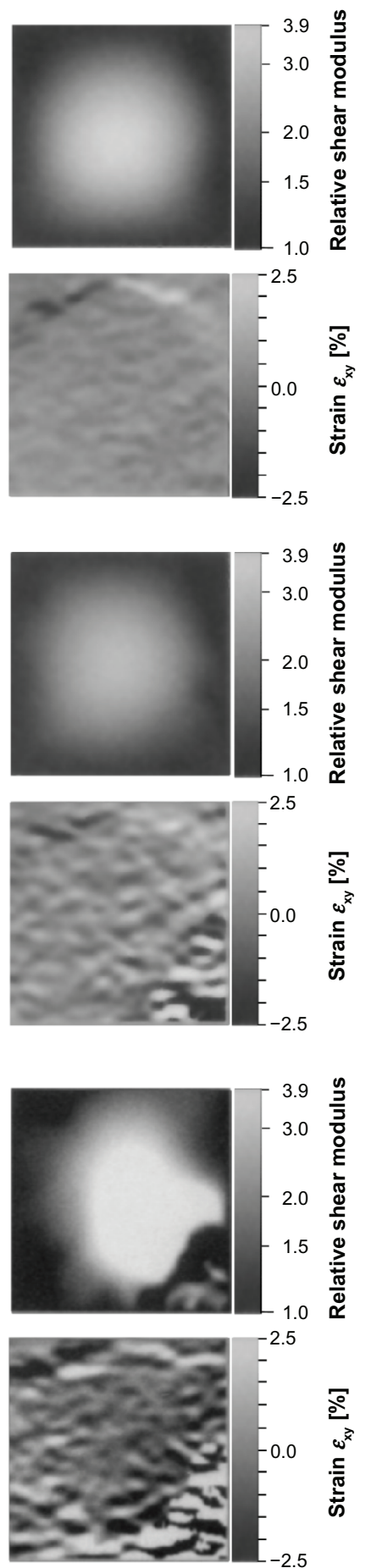

Figure 8 (Continued) 

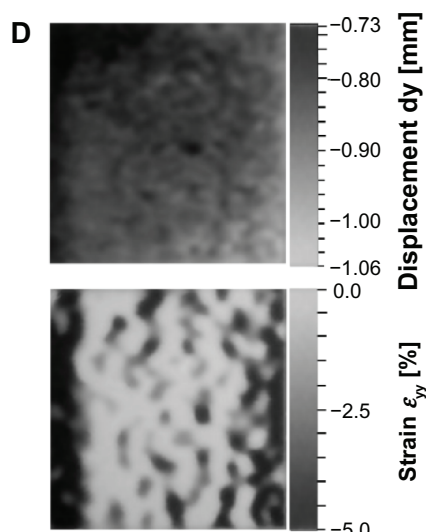

E
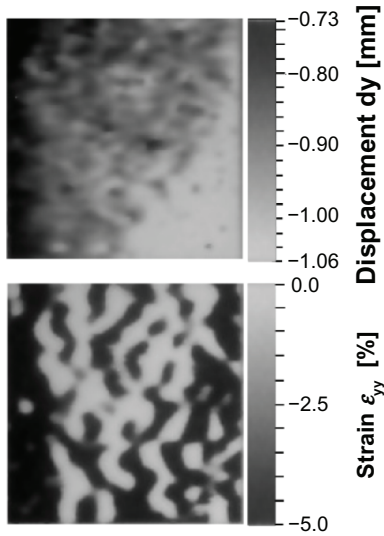
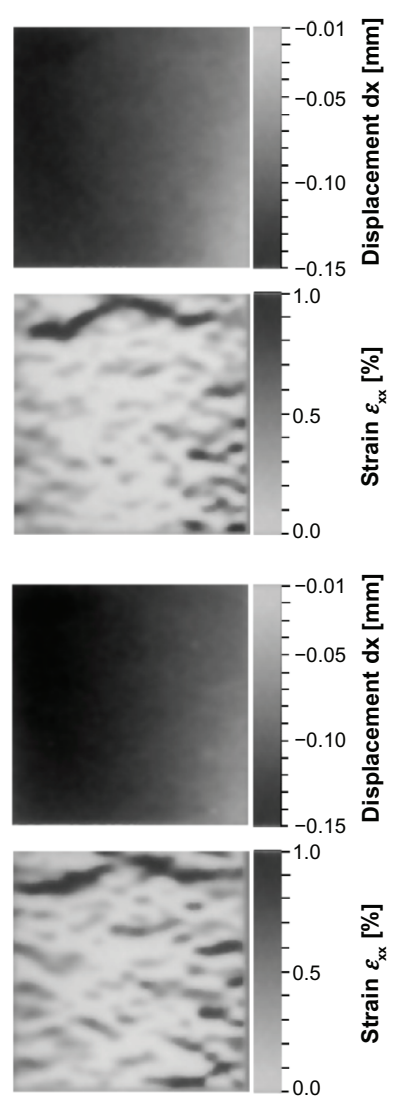
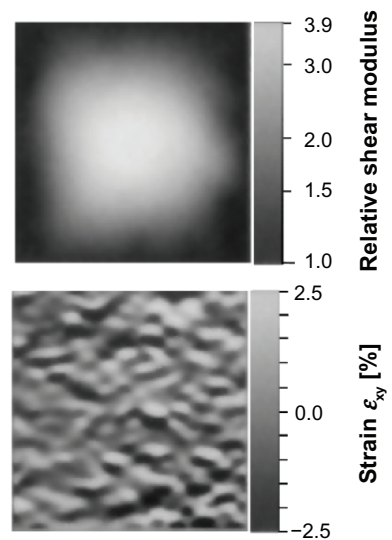

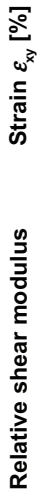

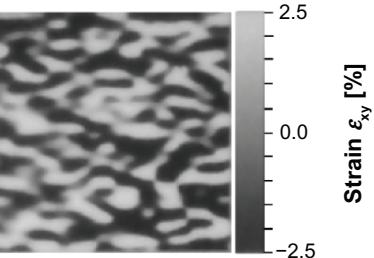

F
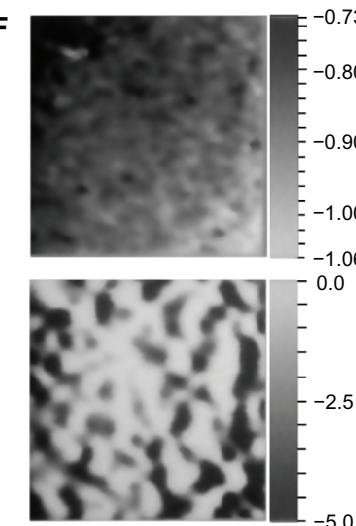

G
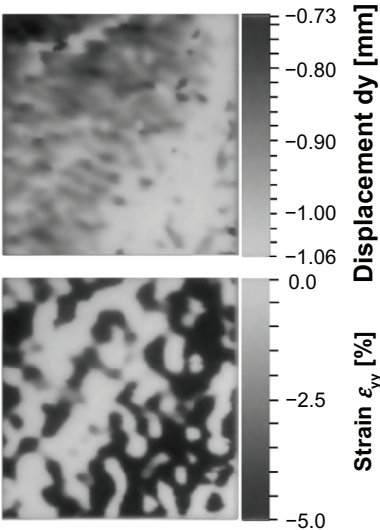
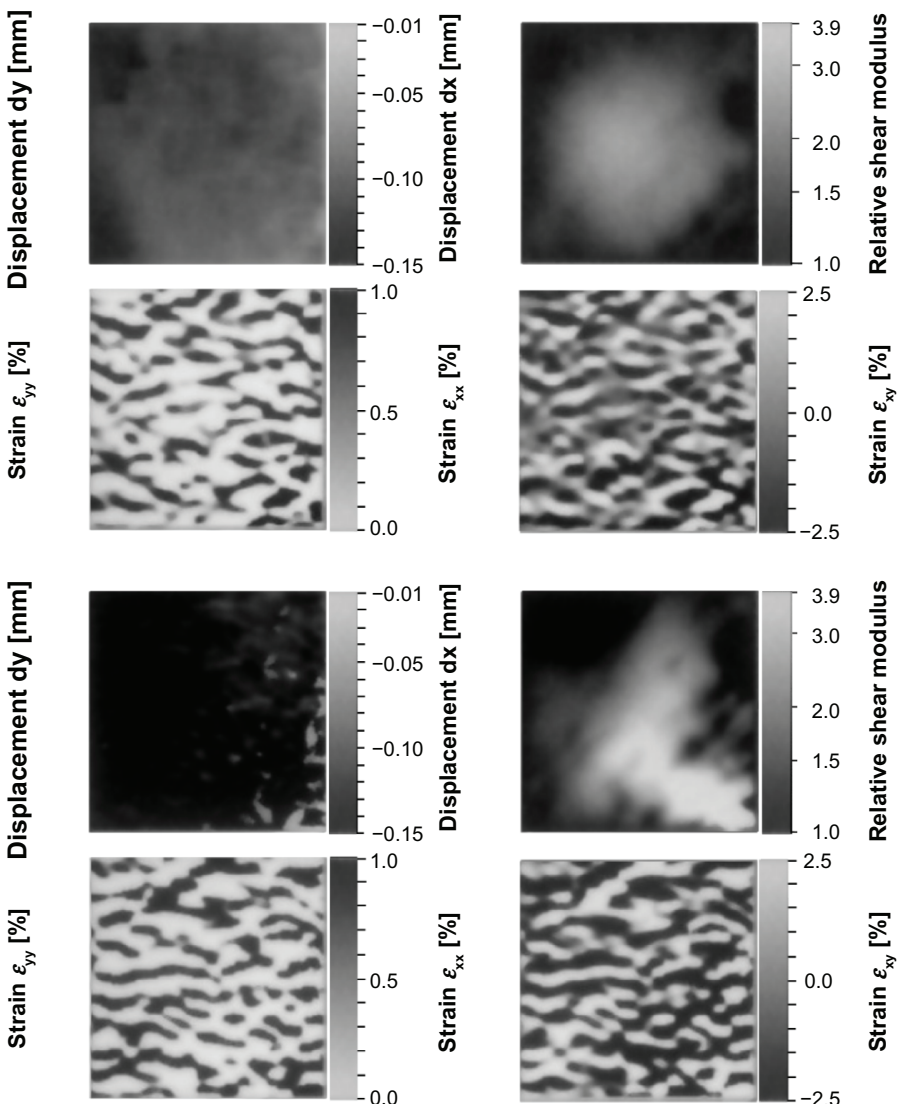

Figure 8 (Continued) 
H
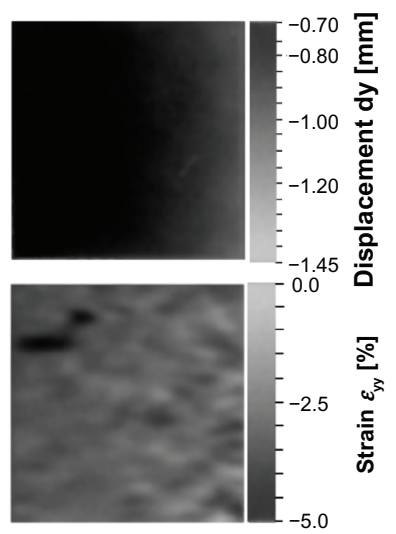

I
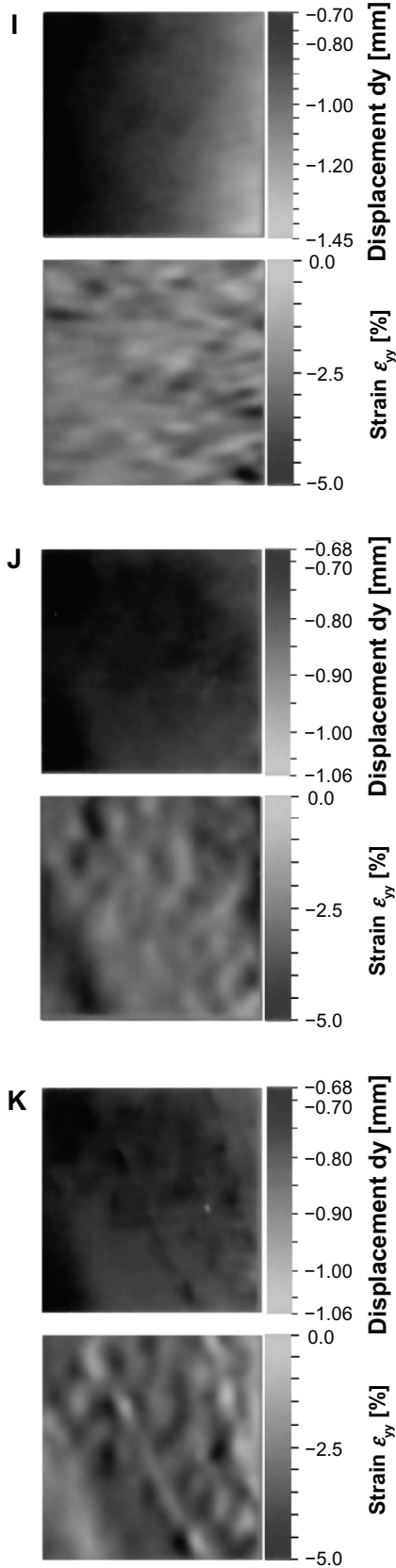
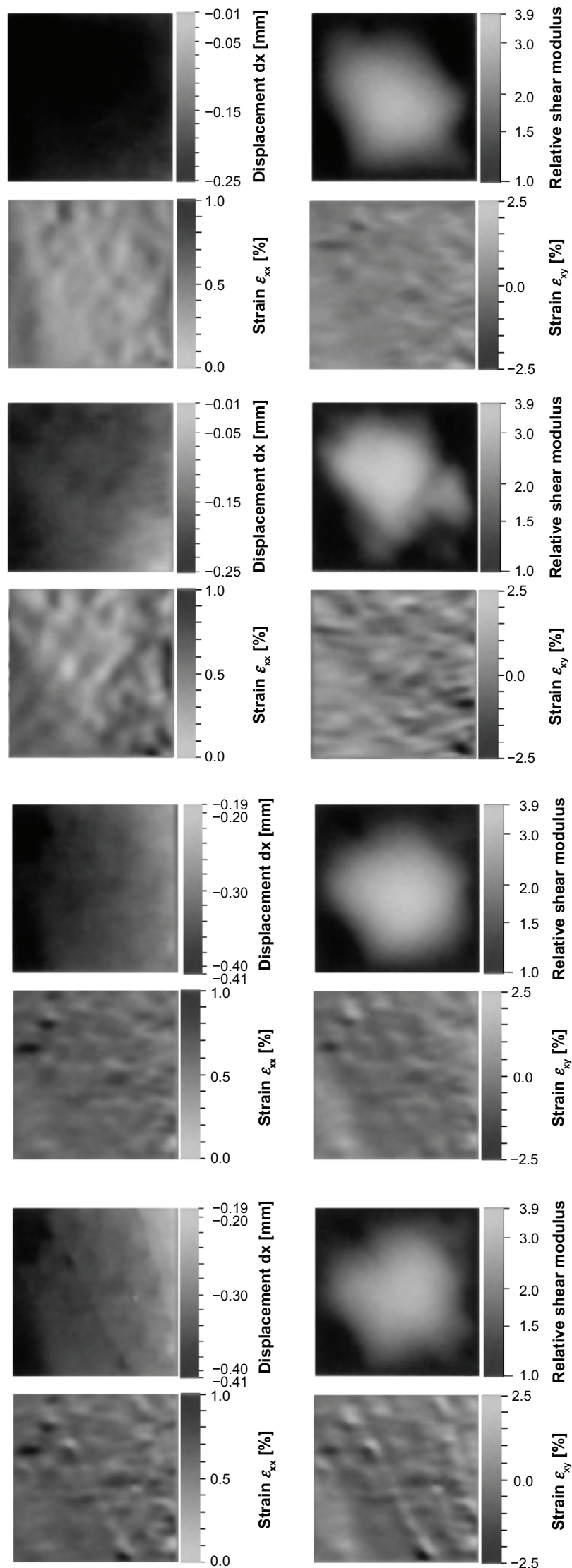

Figure 8 For the same agar phantom compressed in a lateral direction (Figures IA, 4A, and 5A), gray-scale images of the displacement vector (lateral dy and axial dx components), strain tensor (lateral $\varepsilon_{y y}$, axial $\varepsilon_{x x}$, and shear $\varepsilon_{x y}$ ) and the shear modulus measured using the methods $(\mathbf{A})-(\mathbf{K})$ depicted in Figure 7 .

Abbreviation: $\mathrm{mm}$, millimeters. 
SFDM for ASTA, as confirmed by simulations for lateral displacement $\left(\theta=90^{\circ}\right.$, Figure 3B), nonsteering yields more accurate measurements than steering (green circles), ie, steering angles of $0^{\circ}$ (Figures $8 \mathrm{D}$ and $\mathrm{E}$ ) vs $11.9^{\circ}$ ( $8 \mathrm{~F}$ and $\left.\mathrm{G}\right)$. For coordinate rotation (blue circles), as shown in the simulations (Figure 6B), smaller SDs were obtained for $25.9^{\circ}$ (Figures 8J and $\mathrm{K}$ ) than for $11.9^{\circ}$ (Figures $8 \mathrm{H}$ and $\mathrm{I}$ ).

For LM (spherical focusing and steered plane waves, water-blue circles) and the combination of SFDM and coordinate rotations with $11.9^{\circ}$ and $25.9^{\circ}$ (spherical focusing, blue circles), almost the same accuracy was obtained (see also the corresponding images in Figures 8A, B, H, and J). The accuracy obtained with a coordinate rotation of $11.9^{\circ}$ (blue) is higher than that obtained when using steering with the same angle $\left(11.9^{\circ}\right.$, green), and this is likely because the lateral bandwidth is larger, although the axial frequency decreases geometrically (Figures 8F and G vs Figures 8H and I).

In addition, there are significant changes in the accuracy of the measured strains (Figure 7B) and shear moduli (Figure 7C), which are dependent on the beamforming methods and angles used, even though the accuracy was almost same for the displacement vector measurement (see also Figure 8). Accurate means and small SDs were obtained only for LM (Figure 8A) and SFDM using spherical focusing for transmission, and particularly for the nonsteered example $\left(0^{\circ}\right.$, Figure $\left.8 \mathrm{D}\right)$ and the rotation example $\left(25.9^{\circ}\right.$, Figure 8J). However, as shown in the images, except for LM, the geometry of the stiff circular inclusion is distorted.

\section{Lateral displacement measurements}

The accuracies of the previously developed lateral displacement measurement methods ${ }^{17,18}$ such as the mirror setting method and lateral Doppler method using ASTA, were compared with a conventional Doppler method (specifically, a one-dimensional autocorrelation method, but with a twodimensional moving average on an instantaneous ultrasonic frequency and an instantaneous phase change) using ASTA and an angle correction between the directions of the ultrasonic beam and target motion, and the previously developed twodimensional autocorrelation method ${ }^{11-13}$ and one-dimensional autocorrelation method with a demodulation method ${ }^{17,18}$ using LM. Although the demodulation method ${ }^{17,18,31}$ developed by Sumi et al for LM uses only digital signal processing, and is different from other demodulation methods, ${ }^{23,25}$ the onedimensional measurement methods result in lower measurement accuracy than the corresponding multidimensional measurement methods..$^{9,10,13,27}$ Although the one-dimensional measurement methods can be used in place of multidimensional measurement methods, ${ }^{22-25}$ a decorrelation of local echo signals can occur due to target displacement in directions orthogonal to the beams. To cope with this decorrelation, the multidimensional moving average is performed ${ }^{13,17,18,20,29,31}$ (for the multidimensional cross-spectrum phase gradient method, a multidimensional cross-spectrum is calculated using a multidimensional local region).

\section{Simulations}

The same two-dimensional echo data simulated for the displacement vector measurements were used, ie, the same rigid lateral motion $\left(\theta=90^{\circ}\right.$ in Figure $\left.1 \mathrm{~A}\right)$ with a $0.01 \mathrm{~mm}$ magnitude, and steering angles of $\theta=0^{\circ}-80^{\circ}$. By adding white noise, a $20 \mathrm{~dB}$ echo signal-to-noise ratio was also generated. For the conventional Doppler method, the coordinate rotation was performed to set the generated beams in the axial direction $\left(\theta=0^{\circ}\right.$ in Figure 1A).

Figure 9 shows the statistics of the measured lateral displacements. For all the methods, unbiased measurements were achieved over the range of steering angles used. For a nonnoise-filled case, the order of the accuracy (SD) is, the mirror setting $<\mathrm{LM}<$ demodulation $<$ lateral Doppler $<$ conventional method, whereas for the $20 \mathrm{~dB}$ echo signal-to-noise ratio case, the order of accuracy is the mirror setting $<\mathrm{LM}<$ lateral Doppler $<$ conventional method $<$ demodulation method. When the echo signal-to-noise ratio decreases, the accuracy of the demodulation method decreases significantly. In particular, it is meaningful that for the simulations, the lateral displacement measurement methods yield a higher measurement accuracy than other methods, ie, the conventional Doppler method or LM methods.

\section{Agar phantom experiments}

Next, lateral displacement measurements, except for that using the mirror setting, were made on the same agar phantom. Here, four ASTAs were generated. Because the direction of lateral motion was slanted (a mean of $7.6^{\circ}$ ), with the lateral Doppler method the echo data frames obtained were rotated by $-7.6^{\circ}$ using linear interpolation. For the conventional method, the coordinate rotation was also performed to generate beams in the axial direction. That is, coordinate rotation was not performed during beamforming (synthetic aperture) here. Four steering angles which were generated with respect to the dominant target motion were $4.3^{\circ}, 16.2^{\circ}, 19.5^{\circ}$, and $31.5^{\circ}$.

In addition to the lateral Doppler and conventional methods, SFDM ${ }^{18,28-30}$ was used. Two other versions of SFDM 


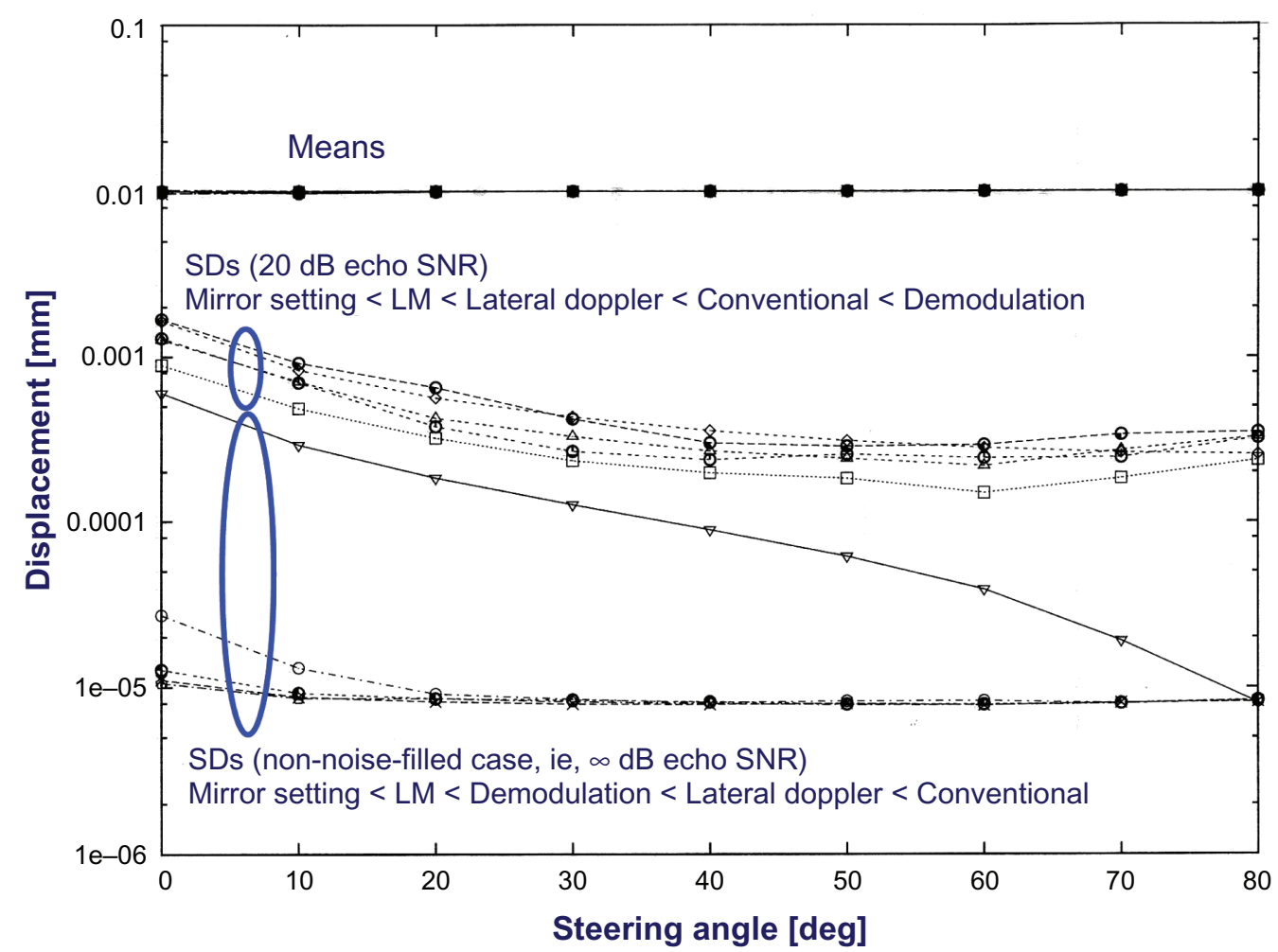

Figure 9 For the same simulated echo data with lateral displacement (magnitude, $0.01 \mathrm{~mm} ; \theta=90^{\circ}$ in Figure IA), an evaluation of the means and standard deviations of the measured lateral displacements.

Abbreviations: $\mathrm{mm}$, millimeters; SDs, standard deviations; dB, decibels; SNR, signal-to-noise ratio; LM, lateral modulation.

were also used. That is, when using the original SFDM, ${ }^{18,28-30}$ displacement in a steered beam direction was synthesized by measuring a displacement vector without coordinate rotation, whereas when using SFDM version 1, the displacement in the steered beam direction was obtained by setting the generated beams in the axial direction by a coordinate rotation similar to the conventional Doppler method used; when using SFDM version 2, the lateral displacement was directly obtained as a lateral displacement component of the measured displacement vector after an angle correction $\left(7.6^{\circ}\right)$ by a coordinate rotation similar to the one used with lateral Doppler. The steering angles were evaluated using the first moments of the spectra.

Figure 10A and B, respectively, show for the five methods, the statistics of the lateral displacements measured for the four steering angles, and the lateral strains measured only for the steering angle $\theta=19.5^{\circ}$ (which corresponds to one of the crossed beams for LM with $\pm 11.9^{\circ}$ ), whereas Figure 11A and B, respectively, show gray-scale images of the lateral displacements and lateral strains (lateral derivatives of the lateral displacements) measured for the steering angle $\theta=19.5^{\circ}$. As shown, increasing the steering angle increases all of the measurement accuracies. Except for SFDM version 2, the order of measurement accuracy (SD) is
$\mathrm{SFDM} \approx \mathrm{SFDM}$ version $1<$ lateral Doppler $\approx$ conventional method. Theoretically, the accuracies of the lateral Doppler and conventional methods, and those of the original SFDM and SFDM version 1 are respectively same. Also, recall that for a nonsteered case, SFDM yielded a higher accuracy than the conventional method (in simulations ${ }^{13}$ and agar phantom experiments ${ }^{27}$ ). These results can also be confirmed for the steering case here. In terms of real-time processing and the accuracy achieved, the original SFDM method, which does not require a coordinate rotation, is the best of the five methods. Some measurement errors were confirmed for the lateral Doppler measurement (see the corresponding image in Figure 11). The measurement accuracy of SFDM1 is also low, particularly the lateral strain measurement. Also note that for small steering angles, SFDM version 2 yields more accurate measurements than the others. In addition, because the target did not move uniformly in the lateral direction, the lateral measurement methods yield lower accuracies and stabilities than the LM methods, eg, for the lateral displacement measurement and displacement vector magnitude, the means and SDs are 0.84 and 0.023 (Figure 10A) vs 0.84 and $0.010 \mathrm{~mm}$ (Figure 7A); for the lateral strains, -0.018 and 0.0066 (Figure 10B) vs -0.0018 and 0.0027 (Figure 7B). See also the images in Figure 11. 

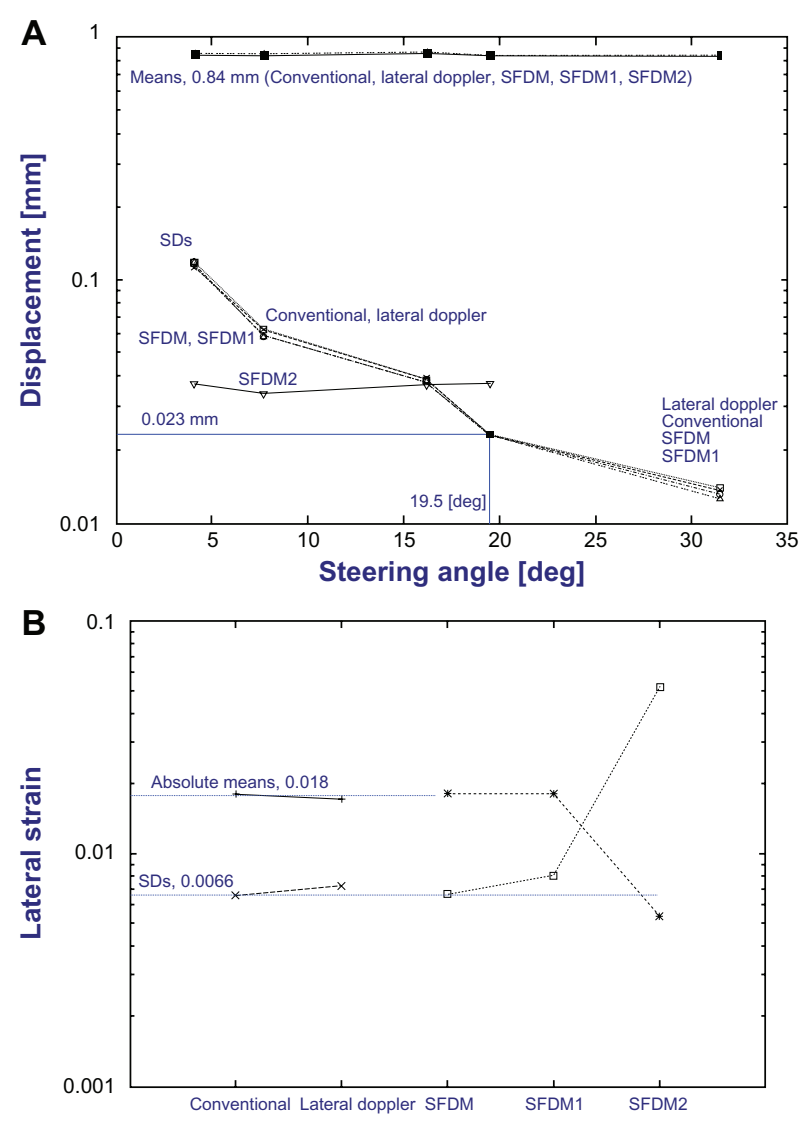

Figure 10 (A) Steering angle vs statistics for lateral displacements measured using a conventional method, lateral Doppler, and versions of the spectra frequency division method, ie, spectra frequency division method (original), spectra frequency division method I and spectra frequency division method 2. (B) For a steering angle of $19.5^{\circ}$, statistics for lateral strains measured using the same methods (absolute means and standard deviations).

Abbreviations: $\mathrm{mm}$, millimeters; SFDM, spectra frequency division method; SDs, standard deviations; deg, degrees.

\section{Discussion}

For measurements of both a displacement vector and a lateral displacement vector using ASTA, SFDM and/or coordinate rotation was more effective in achieving higher accuracy than the block-matching methods previously use ${ }^{18}$ (in simulations, the respective SDs were about 10 and 100 times as large as the pure LM method). It was confirmed that for both LM and ASTA, beams steered in the same direction as the target displacement vectors yield a small SD for the magnitude, and a large SD for the angles. In order to deal simultaneously with the various directions of the displacement vectors, the steering angles should be set at $45^{\circ}$. If the central angles are the same as those of the target displacement vectors, there are small $\mathrm{SD}$ for the magnitude and a large SD for the angles. Also, use of symmetric beams with respect to the axial direction yields accurate measurements. Regarding the coordinate rotation, the rotation angles should be respectively smaller than $10^{\circ}$ and $20^{\circ}$ for $\mathrm{LM}$ with steering angles of $10^{\circ}$ and $20^{\circ}$. The generation of symmetric beams by coordinate rotation is also effective in increasing the accuracy of measurement. For such beams, the one-dimensional measurement method with the newly developed demodulation method can also be used for displacement vector measurements, although the accuracy is lower than for the multidimensional measurement method of LM as shown. ${ }^{31}$ Alternatively, the rotation angle should be set at about $30^{\circ}$ for SFDM.

In the agar phantom experiment, regarding the displacement vector measurement, almost the same results were obtained as those obtained with the simulations shown. The accuracies of strains and shear moduli were also compared. It was confirmed that SFDM achieves almost the same accuracy as the LM method, although only a single steering angle is used. Together with coordinate rotation, nonsteered beam was treated as a version of ASTA ${ }^{28-30}$ (ie, the first version of MAM and MDM without LM). 11,12,14,20,27 Because such a beam yields a larger lateral bandwidth than a steered beam, SFDM becomes more effective. For instance, coordinate rotation with $25.9^{\circ}$ yielded accurate measurements, although the geometry of the stiff circular inclusion was distorted (Figure $8 \mathrm{~J}$ vs LM results, Figure 8A). Alternatively, the use of a plane wave for transmission was effective only for the displacement measurement. Methods using the plane wave will be useful for measurement/imaging of rapid motion or velocity such as blood flow in an artery, or three-dimensional measurement/imaging using a two-dimensional ultrasonic array transducer. Such a laterally wide wave transmission will also be effective when using arbitrary-type array transducers with or without virtual source, ${ }^{32}$ eg, convex, concave, radial types for liver, heart, and blood vessels. In addition, the measurement accuracies evaluated for LM and ASTA will permit finding a proper steering angle and/or a proper rotation angle after coarse measurements of target displacements in order to achieve the highest measurement accuracy. Rotation of the coordinate system will also be effective when obstacles such as bones are encountered.

In this report, only two division spectra were used for a two-dimensional displacement vector measurement. As shown by the agar phantom experiment, vertical and horizontal divisions should be performed for steering angles smaller and larger than $45^{\circ}$, respectively. In this report, the use of an over determined system generated by multiple divisions is also proposed. Various division methods are also described. The point of proper SFDM is to obtain independency of derived equations with a high echo signal-to-noise 

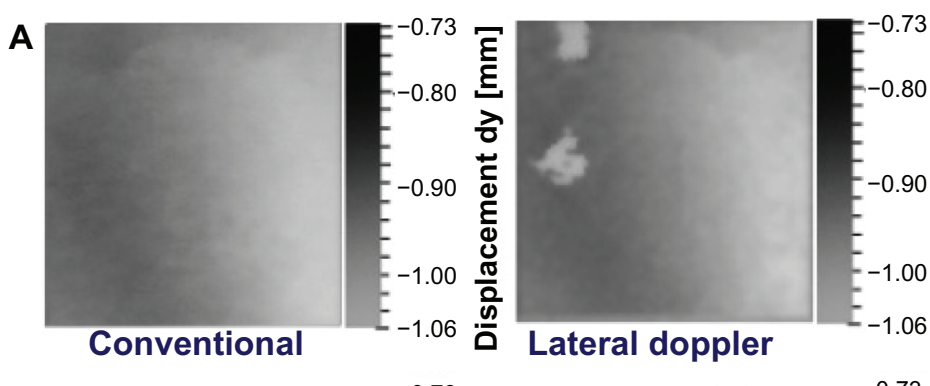

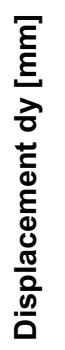
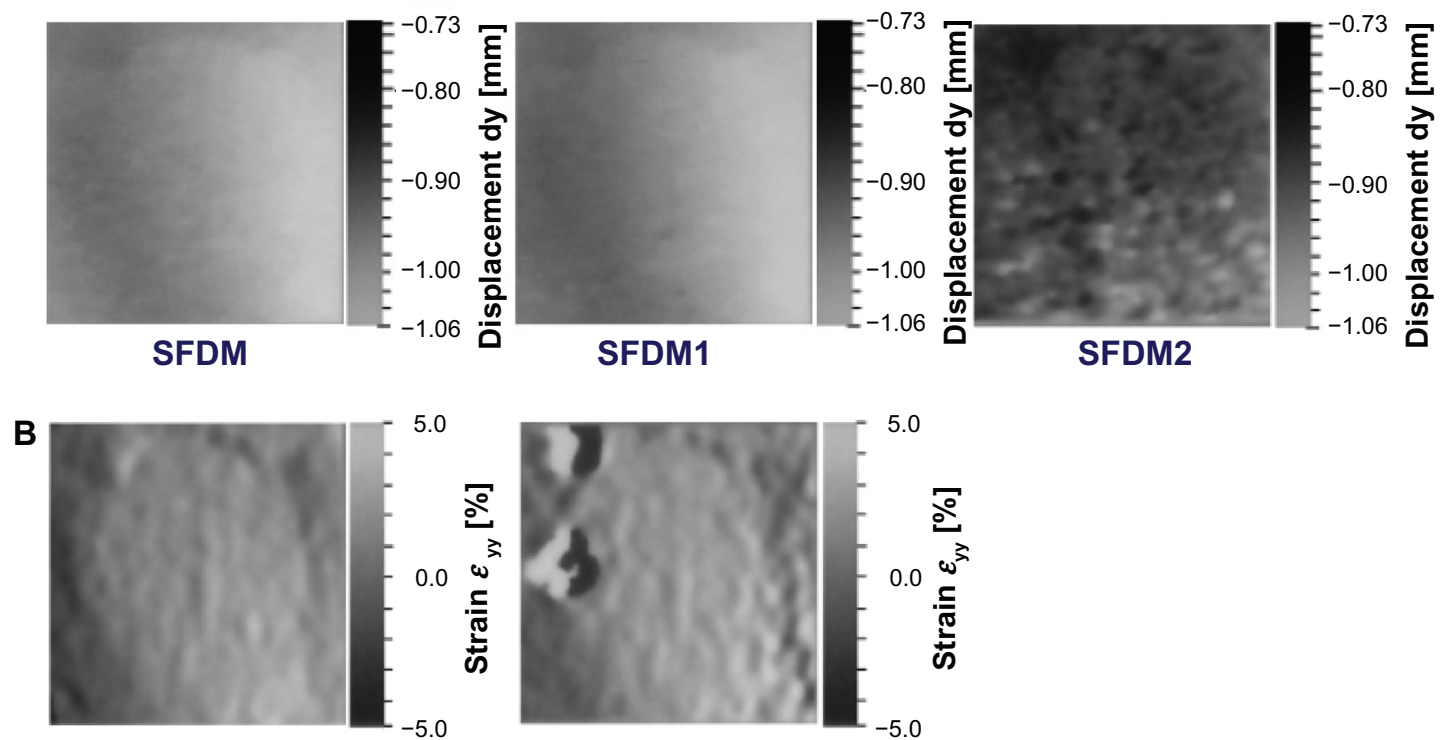

Conventional

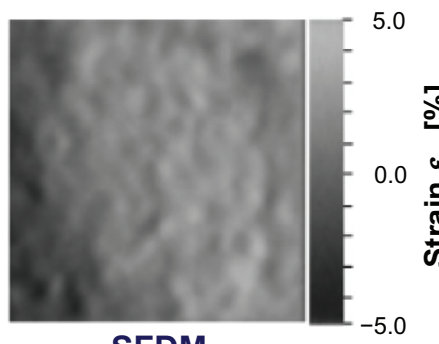

SFDM

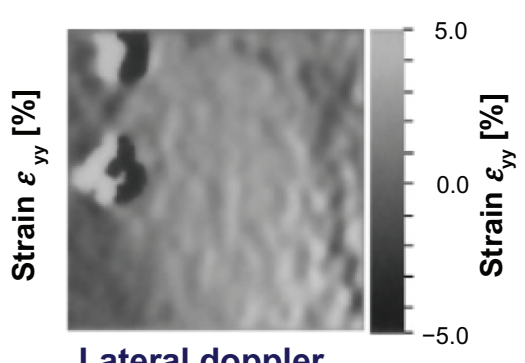

Lateral doppler

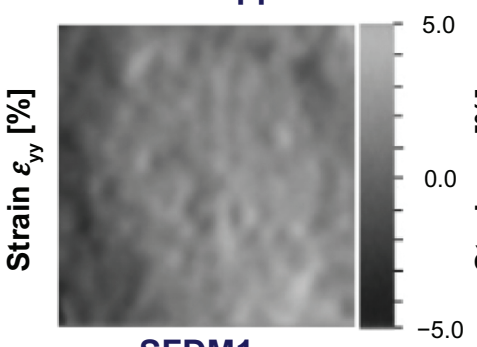

SFDM1

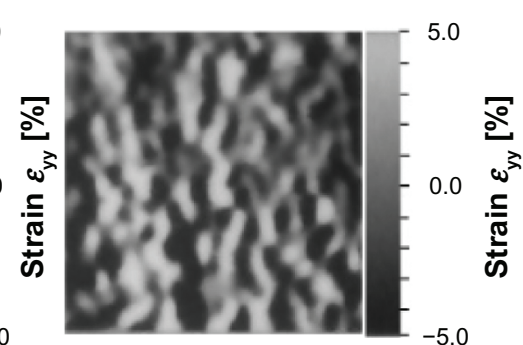

SFDM2

Figure I I For a steering angle of $19.5^{\circ}$, gray-scale images of (A) lateral displacements and (B) lateral strains measured using a conventional method, lateral Doppler, and versions of the spectra frequency division method, ie, spectra frequency division method (original), spectra frequency division method version I and spectra frequency division method version 2 .

Abbreviations: mm, millimeters; SFDM, spectra frequency division method.

ratio and a large bandwidth. As an alternative application for SFDM, disregarding low frequency spectra ${ }^{28-30,33}$ is also effective in the sense that the instantaneous frequency increases, although the bandwidth decreases. For echo imaging, SFDM can also be used for reduction of speckles by superposing echo signals after envelope detection. ${ }^{33}$ When the coordinate rotation increases the axial frequency, the lateral frequency decreases, and vice versa. As explained, the increment and decrement are determined geometrically. However, beam steering (ASTA with nonzero steering) allows maintenance of axial frequency to some extent when the lateral frequency increases, although the echo signal-to-noise ratio and bandwidth decrease. The results of such application will be reported elsewhere with the window used for SFDM.

In this report, lateral displacement measurements were also shown. For the lateral measurement, ASTA can also enable the use of a simpler manual technique with the lateral Doppler method than the conventional Doppler measurement technique (see also Appendix A). It was meaningful that for the simulations, such a lateral displacement measurement method yields a higher measurement accuracy than other methods, ie, the conventional Doppler method or LM methods. Lateral 
displacement measurements were also made on the same agar phantom. Because the direction of lateral motion was slanted (by a mean of $7.6^{\circ}$ ), with the lateral Doppler method the echo data frames obtained were rotated by $-7.6^{\circ}$ using linear interpolation. In addition to the lateral Doppler and conventional methods, several versions of SFDM ${ }^{18,28-30}$ were also used. The steering angle was estimated with high accuracy by estimating the locations of the respective beam centers (ie, the centers of the spectra), ${ }^{18}$ but in practical applications, a sensor for the mechanical steering angle and an automatic detector for blood vessels will also be required. Moreover, being different from the simulation results, the lateral measurements were less accurate than LM measurements due to the practical complex displacements and deformations. Due to the echo interpolation, marked measurement errors were also confirmed for the lateral Doppler measurement, although simpler manual measurement was achieved. Obviously, the echo rotation should be performed at the beamforming.

Another new displacement measurement method was recently developed using an accurate beam angle with a high spatial resolution measured from the first moments of the local spectra or instantaneous frequencies. ${ }^{34}$ These new methods will increase the measurement accuracy of a lateral displacement as well as a displacement vector achieved by synthesizing axial displacements measured from the respective beams with the multiple transmission method or multidirectional synthetic aperture method (ie, nonsuperimposed or separate beams) with a multidimensional moving average, ${ }^{13,17,18,20,21}$ although LM measurement/imaging can also be performed by superimposing the beams. Other groups have also used the multiple transmission method and multidirectional synthetic aperture method with onedimensional methods ${ }^{35-37}$ in such a manner, and except for LM, the beam directions have been determined with delays set to individual array elements. Moreover, although onedimensional measurement methods can be used in place of multidimensional measurement methods, a decorrelation of local echo signals can occur due to target displacement in directions orthogonal to the beams, similar to the LM and demodulation methods. ${ }^{13,17,18,21-25,31}$

Summarizing, a proper beam steering and/or a proper coordinate rotation improves the measurement accuracies of the tissue displacement vector and lateral displacement methods. Such beamforming with the multidimensional displacement vector or lateral displacement measurement method is expected to be used in next-generation ultrasonic imaging equipment. ${ }^{15-18}$ In particular, the LM and ASTA methods will also be used for microscopes, acoustic radiation force imaging, and high intensity focus ultrasound treatment. ${ }^{30}$ However, one should keep in mind that the measurement accuracies obtained with ASTA are lower than with LM. Three-dimensional measurement results will also be reported in the near future.

\section{Disclosure}

The authors report no conflicts of interest in this work.

\section{References}

1. Satomura S. Study of the flow patterns in peripheral arteries by ultrasonics. J Acoust Soc Jpn. 1959;15:151-158.

2. Barker DW. Pulsed ultrasonic Doppler blood-flow sensing. IEEE Trans Sonics Ultrason. 1970;17(3):170-185.

3. Kasai C, Namekawa K, Koyano A, Omoto R. Real-time two-dimensional blood flow imaging using an autocorrelation technique. IEEE Trans Sonics Ultrason. 1985;32(3):458-464.

4. Foster SG, Embree PM, O'Brien WD Jr. Flow velocity profile via time-domain correlation: Error analysis and computer simulation. IEEE Trans Ultrason Ferroelectr Freq Contr. 1990;37(3):164-175.

5. Wilson LS, Robinson DE. Ultrasonic measurement of small displacements and deformations of tissue. Ultrason Imaging. 1982;4(1): 71-82.

6. Ophir J, Cespedes I, Ponnekanti H, Yazdi Y, Li X. Elastography: a quantitative method for measuring the elasticity of biological tissues. Ultrason Imaging. 1991;13(2):111-134.

7. Trahey GE, Allison JW, von Ramm OT. Angle independent ultrasonic detection of blood flow. IEEE Trans Biomed Eng. 1987;34(12): 965-967.

8. Yagi S, Nakayama K. Local displacement analysis of inhomogeneous soft tissue by spatial correlation of rf echo signals. Proceedings of World Federation of Ultrasound in Medicine and Biology. Meeting; Oct 1988; Washington, DC; 113.

9. Sumi C, Suzuki A, Nakayama K. Phantom experiment on estimation of shear modulus distribution in soft tissue from ultrasonic measurement of displacement vector field. IEICE Trans Fundamental. 1995; 78A(12):1655-1664.

10. Sumi C. Fine elasticity imaging on utilizing the iterative rf-echo phase matching method. IEEE Trans Ultrason Ferroelectr Freq Contr. 1999; 46(1):158-166.

11. Sumi C. Digital measurement method of tissue displacement vector from instantaneous phase of ultrasonic echo signal. Technical report of Japan Society of Ultrasound Medicine; 2002; Tokyo, Japan; 37-40.

12. Sumi C. Multidimensional displacement vector measurement methods utilizing instantaneous phase [CD-ROM]. Proceedings of the 27th International Conference IEEE Engineering in Medicine and Biology Society; Sep 2005; Shanghai, China.

13. Sumi C. Displacement vector measurement using instantaneous ultrasound signal phase - multidimensional autocorrelation and Doppler methods. IEEE Trans Ultrason Ferroelectr Freq Contr. 2008; 55(1):24-43.

14. Sumi C. Improvement of measurement accuracy of displacement vector by lateral modulation [in Japanese]. Proceedings of the 2004 Autumn Meeting Acoustical Society of Japan; Sep 2004; Okinawa, Japan; 1353-1354.

15. Sumi C, Noro T, Tanuma A. Effective lateral modulations with applications to shear modulus reconstruction using displacement vector measurement. IEEE Trans on Ultrason Ferroelect Freq Contr. 2008; 55(12):2607-2625. 
16. Sumi C, Tanuma A. Comparison of parabolic and Gaussian lateral cosine modulations in ultrasound imaging, displacement vector measurement, and elasticity measurement. Jpn J Appl Phys. 2008; 47(5B):4137-4144.

17. Sumi C, Matsui N, Shimizu K, Takanashi Y. Preliminary experiments on virtual source for lateral modulation. Proceeding of the 8th International Conference on the Ultrasonic Measurement and Imaging of Tissue Elasticity; September 14-17, 2009; Vlissingen, The Netherlands. Available from: http://www.elasticityconference.org/prior_conf/2009/ PDF/088SumiC02FP.pdf. Accessed September 24, 2011.

18. Sumi C. Lateral displacement measurement using steered beams with a steering angle. Reports in Medical Imaging. 2010;3:61-81.

19. Chen X, Zohdy MJ, Emelianov SY, O’Donnell M. Lateral speckle tracking using synthetic lateral phase. IEEE Trans Ultrason Ferroelectr Freq Contr. 2004;51(5):540-550.

20. Sumi C. Displacement measurement method and apparatus, strain measurement method and apparatus, elasticity and viscoelasticity constants measurement apparatus, and the elasticity and viscoelasticity constants measurement apparatus based treatment apparatus. 4260523 (Japanese Patent) 2009, 7,775,980 B2 (US Patent) 2002 (application).

21. Sumi C. Usefulness of ultrasonic strain measurement-based shear modulus reconstruction for diagnosis and thermal treatment. IEEE Trans Ultrason Ferroelectr Freq Contr. 2005;52(10):1670-1689.

22. Jensen JA. A new method for estimation of velocity vectors. IEEE Trans Ultrason Ferroelectr Freq Contr. 1998;45(3):837-851.

23. Jensen JA. A new estimator for vector velocity estimation. IEEE Trans Ultrason Ferroelectr Freq Contr. 2001;48(4):886-894.

24. Anderson ME. Multi-dimensional velocity estimation with ultrasound using spatial quadrature. IEEE Trans Ultrason Ferroelectr Freq Contr. 1998;45(3):852-861.

25. Anderson ME. A heterodyning demodulation technique for spatial quadrature. Proceeding of the 2000 IEEE Ultrasonics Symposium; October 2000; San-Juan, Puerto Rico; 1487-1490.

26. Sumi C. Relative shear modulus reconstruction for visualization with no geometrical artifact. Acousl Sci Tech. 2010;31(5)347-359.

27. Sumi C, Ebisawa T. Phantom experiments of axial strain measurements using multidimensional autocorrelation method, multidimensional Doppler method and direct strain measurement method. Acoust Sci Tech. 2009;30(2):117-123.
28. Sumi C. Displacement vector measurement using ASTA and spectra division [in Japanese]. Proceedings of the 2010 Autumn Meeting Acoustical Society of Japan; Sep 2010; Osaka, Japan; 3-17.

29. Sumi C, Shimizu K, Takanashi Y. Increase in spatial resolution of lateral modulation imaging. Proceedings of the 9th International Conference of Ultrasonic Measurement and Imaging of Tissue Elasticity; Snowbird, UT; Oct 2010: 71

30. Sumi C, Ichimaru K, Shinozuka Y. Increase in measurement accuracy of tissue displacement vector using rotation of coordinate system [in Japanese]. The Institute of Electronics, Information and Communication Engineers. US2010-82, 2010:25-32.

31. Sumi C, Shimizu K. Ultrasonic agar phantom experiment for comparison of the measurement accuracy of tissue elasticity obtained by displacement vector measurement using lateral modulation with multidimensional autocorrelation and Doppler methods and corresponding one-dimensional methods. Reports in Medical Imaging. 2010;4:39-46

32. Sumi C. Effective ultrasonic virtual sources which can be positioned independently of physical aperture focus position. Reports in Medical Imaging. 2010;3:45-59.

33. Sumi C. Multidimensional ultrasonic imaging and displacement vector measurement using spectra frequency division method [in Japanese]. The Institute of Electronics, Information and Communication Engineers. 2011:US-07:85-90.

34. Sumi C. Increase in accuracy of echo imaging and displacement measurement by considering the direction of beam-steering application to lateral modulation (LM) and a steering angle (ASTA) [in Japanese]. The Institute of Electronics, Information and Communication Engineers. 2011:US-05:1-8.

35. Fox MD. Multiple crossed-beam ultrasound Doppler velocimetry. IEEE Trans Ultrason Ferroelectr Freq Contr. 1978;25(5):281-286.

36. Techavipoo U, Chen Q, Varghese T, Zagzebski JA. Estimation of displacement vectors and strain tensors in elastography using angular isonifications. IEEE Trans Med Imaging. 2004;23(12):1479-1489.

37. Tanter M, Bercoff J, Sandrin L, Fink M. Ultrafast compound imaging for 2-D motion vector estimation: application to transient Elastography. IEEE Trans Ultrason Ferroelectr Freq Contr. 2002; 49(10):1363-1374. 


\section{Appendix A}

\section{Advantages of ASTA with respect to lateral modulation}

Lateral modulation uses two steering angles for twodimensional measurement/imaging, and three or four steering angles for three-dimensional measurement/imaging, ie, multiple crossed beams. For a two-dimensional case, if a beam is transmitted with a ultrasonic frequency, $\mathrm{f}_{0}$, in the direction expressed using a steering angle $\theta$ (Figure 1A), axial (x) and lateral (y) frequencies are expressed, respectively, as: ${ }^{18}$

$$
\mathrm{f}_{\mathrm{x}}=\mathrm{f}_{0} \cos \theta \text { and } \mathrm{f}_{\mathrm{y}}=\mathrm{f}_{0} \sin \theta
$$

Then, if a laterally symmetric beam with the same ultrasonic frequency, $\mathrm{f}_{0}$, is also generated and crossed at position $(\mathrm{x}, \mathrm{y})$, a ultrasonic echo signal from the position can be expressed as:

$\mathrm{A}(\mathrm{x}, \mathrm{y}) \cos 2 \pi \frac{2}{\lambda}(\mathrm{x} \cos \theta+\mathrm{y} \sin \theta)$

$+\mathrm{A}^{\mathrm{\prime}}(\mathrm{x}, \mathrm{y}) \cos 2 \pi \frac{2}{\lambda}(\mathrm{x} \cos \theta-\mathrm{y} \sin \theta)$

where $\mathrm{A}(\mathrm{x}, \mathrm{y})$ and $\mathrm{A}^{\prime}(\mathrm{x}, \mathrm{y})$ are, respectively, amplitudes of echo signals from the position obtained by the two steered beams, and $\lambda$ is a wavelength.

Here, if $\mathrm{A}(\mathrm{x}, \mathrm{y})=\mathrm{A}^{\prime}(\mathrm{x}, \mathrm{y})$ and $\mathrm{A}(\mathrm{x}, \mathrm{y})=-\mathrm{A}^{\prime}(\mathrm{x}, \mathrm{y})$ are respectively realized by even and odd apodizations, the correspondingly received echo signals can be expressed as laterally cosine and sine modulated signals as follows:

and

$$
2 \mathrm{~A}^{\prime}(\mathrm{x}, \mathrm{y}) \sin 2 \pi \frac{2 \cos \theta}{\lambda} \mathrm{x} \sin 2 \pi \frac{2 \sin \theta}{\lambda} \mathrm{y} .
$$

\section{Publish your work in this journal}

Reports in Medical Imaging is an international, peer-reviewed, open access journal publishing original research, reports, reviews and commentaries on all areas of medical imaging. The manuscript management system is completely online and includes a very quick and fair peer-review system, which is all easy to use.

As mathematically expressed, an increase in the steering angles increases the lateral frequency $f_{y}$, and vice versa. That is, the increment and decrement are determined geometrically. However, in real-world applications, beam nonzero steering allows maintenance of the axial frequency to some extent when the lateral frequency increases, although the echo signal-to-noise ratio and bandwidth decrease.

Alternatively, ASTA is simpler than lateral modulation, ${ }^{17,18}$ which uses only a steering angle. All of the beamforming methods, including ASTA, can be performed on an arbitrary orthogonal coordinate system. However, ASTA has several advantages with respect to lateral modulation. ${ }^{17,18}$ For instance, (i) at the minimum, fewer calculations are required to complete beamforming than with lateral modulation, the multiple transmission method, and multidirectional synthetic aperture method; (ii) effects caused by the existence of obstacles, such as bone, decrease because a smaller physical aperture can be used; (iii) an inhomogeneity in tissue properties, such as propagation speed, does not affect focusing (ie, the beam-crossing position) because multiple beams which have different paths are not used; (iv) tissue motion artifacts which can occur with lateral modulation when using plural transmitted beams with a time difference are not generated; (v) in conjunction with (iv), a higher echo signalto-noise ratio can be obtained than with lateral modulation when lateral modulation uses a classical synthetic aperture.

Submit your manuscript here: http://www.dovepress.com/reports-in-medical-imaging-journal

Visit http://www.dovepress.com/testimonials.php to read real quotes from published authors. 